\title{
A Hybrid Precipitation Index Inspired by the SPI, PDSI, and MCDI. Part II: Application to Investigate Precipitation Variability along the West Coast of North America
}

\author{
Dudley B. Chelton And Craig M. Risien \\ College of Earth, Ocean and Atmospheric Sciences, Oregon State University, Corvallis, Oregon
}

(Manuscript received 25 September 2019, in final form 15 July 2020)

\begin{abstract}
The hybrid precipitation index developed in Part I of this study is applied to investigate precipitation variability along the west coast of North America during the wet season November-March on monthly-to-interannual time scales. The variability in each of six regions considered in this study is negatively correlated with nearby $500-\mathrm{hPa}$ geopotential height anomalies. Except in Southeast Alaska, these correlation patterns indicate that precipitation variability in each region is predominantly influenced by local atmospheric forcing analogous to the ridging of the westerly flow that has been studied extensively with regard to California drought variability. The first empirical orthogonal function (EOF) accounts for nearly all of the Southeast Alaska precipitation variability, which is controlled by the strength of the onshore flow rather than ridging. In association with this mode of variability, precipitation anomalies of opposite sign account for about $40 \%$ of the precipitation variance in Northern California and Oregon on all time scales. On short time scales, the second and third EOFs account primarily for precipitation variability in British Columbia/Washington and California, respectively. With increasing time scale, the third EOF diminishes in importance and the second EOF evolves into a pattern of synchronous precipitation anomalies of the same sign from British Columbia to Northern California. Precipitation variability in Southern California is only modestly related to precipitation elsewhere. With increasing time scale, Southern California precipitation variability becomes increasingly related to precipitation anomalies of opposite sign in Washington.
\end{abstract}

\section{Introduction}

In a companion paper (Chelton and Risien 2020, hereafter Part I), we conducted detailed analyses of the Palmer drought severity index (PDSI) and the standardized precipitation index (SPI), which are the two most commonly used indices for studies of drought and precipitation variability. We also examined the recently developed model calibrated drought index (MCDI). As discussed at length in Part I and detailed further in this study, all three of these drought indices have issues that limit and, in the cases of the PDSI and SPI, complicate their interpretations as indicators of climate variability. This motivated the development of a new hybrid index that retains the strengths of the PDSI, MCDI, and SPI while addressing their various limitations and interpretational issues. This hybrid index can be simply calculated for any region in the world. The objective of this study is to demonstrate the utility of the hybrid precipitation index from an analysis of the geographical patterns

\footnotetext{
Corresponding author: Dudley B. Chelton, chelton@coas oregonstate.edu
}

of precipitation anomalies on time scales ranging from monthly to interannual in six regions along the west coast of North America.

The PDSI (Palmer 1965; Alley 1984) and MCDI (Williams et al. 2017) incorporate the effects of precipitation, evapotranspiration, and runoff. Their use for studies of geographical patterns of drought variability is limited by the fact that they capture a single "intrinsic" time scale of long-term drought variability that varies from region to region. It is shown in Part I that PDSI and MCDI time series consist essentially of exponentially weighted averages of present and past precipitation anomalies. The PDSI also depends weakly on future precipitation because of a complication of the PDSI algorithm referred to as the "backtracking procedure" that is specific to the original intended purpose of the PDSI as an indicator of the beginning and ending of dry or wet spells. The $e$-folding rates determined by the

Publisher's Note: This article was revised on 31 August 2020 to correct two minor typographical errors that appeared in the Introduction when originally published. 
algorithms for the PDSI and MCDI are regionally specific and the resulting time scales can differ between the two indices for any particular region. Because the time scales captured by the PDSI or MCDI cannot be controlled by the user, neither of these drought indices is able to provide insight into drought variability on time scales other than the time scale chosen by each algorithm. Moreover, neither of these indices is available outside of the conterminous lower 48 states of the United States. Furthermore, there are disconcerting artifacts in the PDSI that complicate its interpretation as a drought index (see Part I).

The SPI (McKee et al. 1993; Edwards and McKee 1997) is a precipitation-only index of drought variability ${ }^{1}$ that addresses the limitations of the PDSI and MCDI by allowing users to compute an index for any specified time scale in any region. The SPI algorithm applies a different normalization for each of the 12 calendar months. It is shown in section 2 of Part I that this complicates the interpretation of the SPI for studies of precipitation variability on time scales shorter than about 10 months for any particular region (see also appendix B of this study). For longer averaging time scales, the SPI is essentially equivalent to a block average of past precipitation over the time period specified by the user. These block averages are very simply obtained without the computational complexities of the SPI algorithm. Aside from the well-known undesirable filtering properties of block averages (see Fig. 8 of Part I), uniform weighting of past precipitation is less physical than the implicit exponential weighting of the PDSI and MCDI.

In addition to interpretational complications when analyzing the SPI for a single region that arise from the above-noted normalization procedure, use of the SPI for studies of precipitation covariability in multiple regions is complicated by the fact that the normalization procedure removes all information about the actual precipitation amounts. It is therefore not possible to infer the relative amounts of anomalous accumulated precipitation from the SPI time series for different regions. It is only possible to assess the relative degrees to which the anomalous accumulated precipitation was unusual in each individual region compared with the historical statistics for that region. This interpretational issue applies

\footnotetext{
${ }^{1}$ A standardized precipitation evapotranspiration index (SPEI) has been developed that modifies the SPI to include effects of evapotranspiration that are parameterized based on air temperature and latitude (Vicente-Serrano et al. 2010; Beguería et al. 2014). Correlations between the SPEI and the SPI for a given region typically exceed 0.95 (Part I), indicating that the parameterized evapotranspiration effects contribute little to the SPEI time series beyond what is already accounted for implicitly by precipitation alone.
}

on all time scales, not just on the above-noted time scales shorter than about 10 months on which interpretation of the SPI is complicated in any individual region where there are strong seasonal variations of the statistics of precipitation variability. These interpretational issues with the SPI are discussed in the context of examples in section 2 of Part I and in section 4 and appendix B of this study.

Inspired by the physical basis for the PDSI and MCDI and the flexibility of the SPI for studying precipitation variability on different time scales, a hybrid precipitation index that is easily computed as exponentially weighted averages of present and past precipitation was developed in Part I. MATLAB and Python codes for computing the hybrid index from any monthly averaged time series of precipitation anomalies can be obtained from the GitHub repository as described in appendix A of Part I. The hybrid index is applied in two examples in this study. The application in section 2 uses the exponential weighting that is explicit in the hybrid index and implicit in the PDSI and MCDI to inform the choice of filtering to be applied to atmospheric forcing in order to investigate cause and effect relationships from the PDSI, MCDI, or hybrid precipitation index. The application in section 3 addresses the issue of the geographically varying and nonadjustable filtering properties of the PDSI and MCDI, as well as the lack of availability of both of these indices for regions outside of the lower 48 states of the United States. Specifically, the hybrid algorithm is applied in section 3 to derive precipitation indices with eight different choices of filtering in six regions along the west coast of North America from Southeast Alaska to Southern California to investigate the dependence of geographical patterns of drought and precipitation variations on time scales ranging from monthly to interannual.

It should be noted that the PDSI was developed by Palmer (1965) for the purposes of identifying the beginning, ending and severity of dry and wet spells. While it was not originally intended to be analyzed as a time series indicator of climate variability, it has nonetheless been used for such purposes in many past studies. The analyses in Part I and in section 2 below provide useful context for such applications by quantifying the filtering properties of the PDSI as a time series index of precipitation variability.

The sources for the data used throughout this study are given in appendix A.

\section{Atmospheric forcing of long-term drought variability in California}

The recent 2011-16 drought in California was unusual in its severity and persistence (see, e.g., Swain et al.2017, 
and references therein). Although the mechanism for California droughts remains elusive, the proximate cause of precipitation variability in California has long been understood (e.g., Mitchell and Blier 1997; Ellis and Barton 2012; Wang et al. 2014; Seager et al. 2015; and many others). Drought conditions occur from high pressure anomalies off the coast of Northern California, which cause a ridging of the jet stream that deflects moisture-laden storms to the north. Conversely, wet conditions occur from low pressure anomalies off the coast of Northern California and an associated southward deflection of the jet stream. This negative correlation between monthly anomalies of precipitation and atmospheric pressure is readily apparent from the map in Fig. 1a. The lagged correlations ${ }^{2}$ between Northern California (N.CA) precipitation and 500-hPa geopotential height anomalies at the $42.5^{\circ} \mathrm{N}, 127.5^{\circ} \mathrm{W}$ location of the extremum correlation are shown in Fig. 1b. The extremum correlation of -0.74 occurs at lag zero. There is no significant asymmetry between positive and negative lags and very little persistence; the correlations decrease to -0.15 at lags of \pm 1 month and fluctuate within the range \pm 0.10 at longer lags.

As the PDSI that is examined in detail in section 3 of Part I is specifically intended to be a metric for long-term drought variability, it is an obvious candidate index for characterizing the statistical properties of drought variations with the long time scale of the 2011-16 drought in California. The expected inverse relationship between atmospheric pressure anomalies and the N.CA PDSI estimated as described in appendix $A$ is evident from the map in Fig. 1c of the correlations between gridded values of unsmoothed monthly averaged $500-\mathrm{hPa}$ geopotential height anomalies and the concurrent monthly PDSI. An extremum correlation of -0.41 occurs at the same $42.5^{\circ} \mathrm{N}, 127.5^{\circ} \mathrm{W}$ location of the much higher extremum correlation of -0.74 between unsmoothed monthly anomalies of precipitation and $500-\mathrm{hPa}$ geopotential height in Fig. 1a. A visual examination of the time series at this location (not shown here) reveals that the 500-hPa height anomalies contain much more highfrequency variability than the PDSI time series. This is not surprising since the PDSI is an index of low-frequency drought variability, whereas monthly anomalies of 500-hPa geopotential height have very short time scales. The 500-hPa height time series should therefore be smoothed

\footnotetext{
${ }^{2}$ The $95 \%$ significance levels of the correlations presented throughout this study were estimated by the method of Davis (1977) and Chelton (1983) from long-lag correlations averaged over the range from $40 \%$ to $80 \%$ of the record length. This method has been shown by Michaelsen (1987) to yield results essentially the same as estimates obtained by cross validation.
}

in a manner that is commensurate with the low-frequency time scales of drought variability that are captured by the PDSI.

The structure of the lagged correlations between the N.CA PDSI and unsmoothed 500-hPa geopotential height anomalies at $42.5^{\circ} \mathrm{N}, 127.5^{\circ} \mathrm{W}$ shown in Fig. $1 \mathrm{~d}$ provides insight into the most appropriate form of smoothing that should be applied to the 500-hPa height anomalies. The distinctive asymmetry of the lagged correlations has the same telltale structure as the lagged correlations between the N.CA PDSI and monthly precipitation anomalies in Fig. 10 of Part I, except with opposite signs of the correlations. The intuitively obvious conclusion is that the N.CA PDSI is related to the integrated effects of preceding atmospheric forcing rather than just to concurrent atmospheric forcing. In particular, the analysis of the N.CA PDSI in section $3 \mathrm{~b}$ of Part I and the structure of the lagged correlations in Fig. 1d suggest that the N.CA PDSI is likely most closely related to one-sided, exponentially weighted averages of present and past 500-hPa pressure anomalies with the same exponential time scale of $\tau=6.1$ months that characterizes the relationship of the N.CA PDSI to present and past precipitation in Fig. 10 of Part I.

A map of the correlations between the concurrent monthly PDSI and gridded fields of one-sided, exponentially weighted averages of monthly $500-\mathrm{hPa}$ geopotential height anomalies with the $e$-folding time of $\tau=6.1$ months is shown in Fig. 1e. An extremum correlation of -0.73 occurs at the same $42.5^{\circ} \mathrm{N}, 127.5^{\circ} \mathrm{W}$ location as the extremum correlations in Figs. 1a and 1c. This correlation is much larger in magnitude than the extremum correlation of -0.41 between the PDSI and unsmoothed 500-hPa height anomalies in Figs. 1c and $1 \mathrm{~d}$. The extremum correlation of -0.73 is also larger in magnitude than the correlations between the PDSI and $500-\mathrm{hPa}$ height anomalies smoothed with values of $\tau$ smaller and larger than $\tau=6.1$ months, thus confirming that $\tau=6.1$ months is indeed the appropriate choice of time scale for the exponentially weighted averaging of the 500-hPa height fields that force the precipitation variability represented by the N.CA PDSI.

A close inspection of the lagged correlations between the PDSI and the smoothed 500-hPa height anomalies (the black line in Fig. 1f) reveals that the correlations are not quite symmetric about zero lag. The somewhat higher correlation magnitudes at positive lags than at negative lags could be anticipated from the analysis in section $3 \mathrm{~b}$ of Part I that showed that the backtracking procedure of the PDSI algorithm implicitly includes small contributions from future precipitation values. The $\beta$ parameter that defines the ratio of future to past precipitation in the two-sided, exponentially weighted 
a)

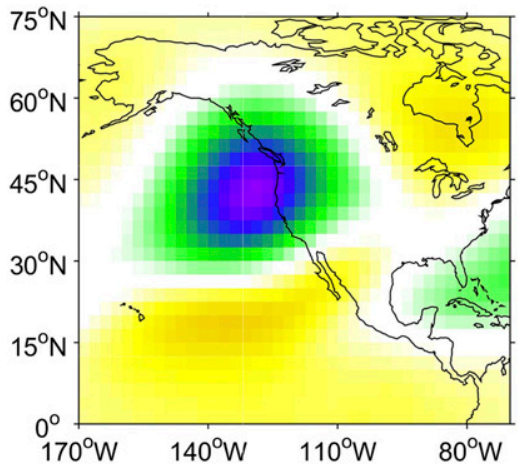

C)

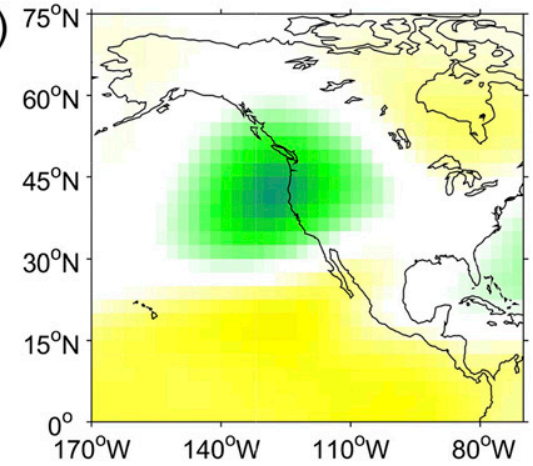

e)

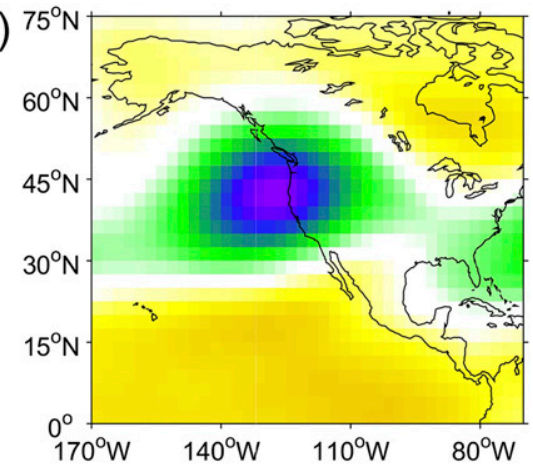

0.8 b)
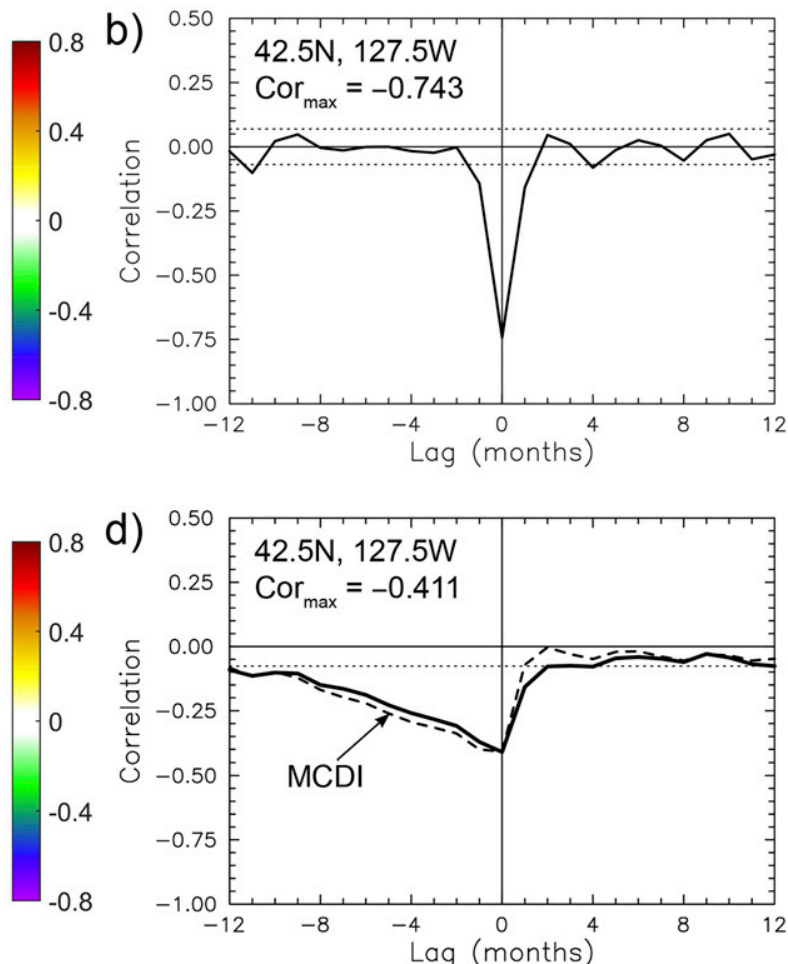

d)

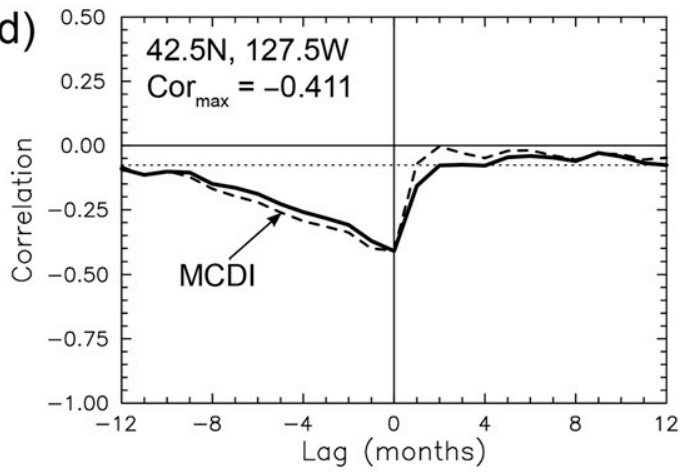

$0.8 \mathrm{f})$

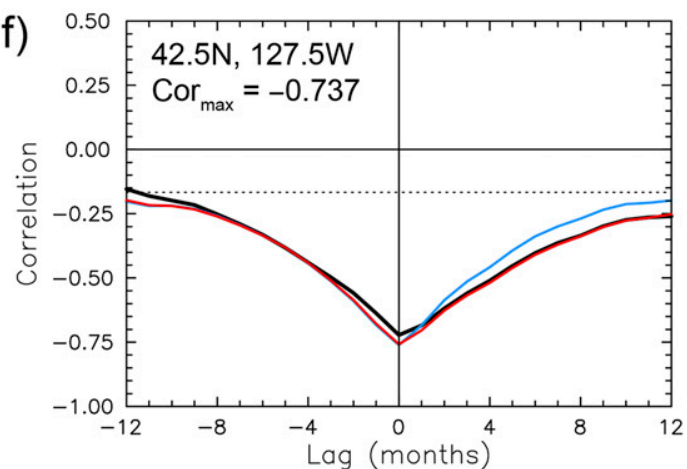

FIG. 1. Correlations between N.CA precipitation anomalies (PCP) and 500-hPa geopotential height anomalies (500HGT) with various combinations of smoothing. (a) Map of the correlations between unsmoothed N.CA PCP and concurrent unsmoothed $500 \mathrm{HGT}$ on a $2.5^{\circ} \times 2.5^{\circ}$ grid. (b) Lagged correlations between unsmoothed N.CA PCP at time $t$ and $500 \mathrm{HGT}$ at $42.5^{\circ} \mathrm{N}, 127.5^{\circ} \mathrm{W}$ at time $t+$ lag. (c) As in (a), but for the correlations between the N.CA PDSI and concurrent unsmoothed 500HGT. (d) As in (b), but for the lagged correlations between the N.CA PDSI at time $t$ and unsmoothed $500 \mathrm{HGT}$ at $42.5^{\circ} \mathrm{N}, 127.5^{\circ} \mathrm{W}$ at time $t+$ lag (solid line). The dashed line is the lagged correlations for the N.CA MCDI. (e) As in (c), but for the correlations of the N.CA PDSI with onesided, exponentially weighted averages of 500HGT with an $e$-folding time of $\tau=6.1$ months. (f) As in (d), but for the lagged correlations between the N.CA PDSI at time $t$ and one-sided, exponentially weighted averages of $500 \mathrm{HGT}$ with an $e$-folding time of $\tau=6.1$ months at $42.5^{\circ} \mathrm{N}, 127.5^{\circ} \mathrm{W}$ at time $t+$ lag (black line). The blue line corresponds to the lagged correlations between the smoothed 500HGT and a hybrid precipitation index defined to be one-sided, exponentially weighted averages of precipitation with an $e$-folding time of $\tau=6.1$ months. The red line is the lagged correlations for a hybrid precipitation index defined to be two-sided, exponentially weighted averages of precipitation with an exponential time scale of $\tau=6.1$ months and a ratio of $\beta=0.15$ for the weighting of future vs past values of precipitation. The dotted lines in the right panels are the estimated $95 \%$ significance levels of the correlations. 
average simulation of the N.CA PDSI in section $3 \mathrm{~b}$ of Part I was estimated to be 0.15 (see Fig. 10 of Part I), indicating that future precipitation is weighted only $15 \%$ as heavily as past precipitation in the PDSI algorithm for N.CA.

The speculation that the small asymmetry of the lagged correlations between the PDSI and one-sided, exponentially weighted averages of $500-\mathrm{hPa}$ height anomalies is attributable to the implicit inclusion of effects of future precipitation anomalies in the PDSI is supported by the blue and red lines in Fig. 1f. The blue line corresponds to the lagged correlations between the smoothed 500-hPa height anomalies and the hybrid precipitation index that consists of one-sided, exponentially weighted averages of precipitation anomalies with the same exponential time scale of $\tau=6.1$ months and $\beta=0$. These lagged correlations are almost imperceptibly different from exact symmetry about zero lag. The extremum correlation of -0.76 is slightly larger in magnitude than the extremum correlation of -0.73 with the PDSI. The red line in Fig. 1f corresponds to the lagged correlations between the smoothed 500-hPa height anomalies and a two-sided, exponentially weighted average precipitation index with the same exponential time scale of $\tau=6.1$ months but with the above-noted value of $\beta=0.15$ that was determined for the N.CA PDSI in Fig. 10 of Part I. For negative and zero lags, the lagged correlations shown by the red and the blue lines are almost exactly the same. For positive lags, however, the red line almost exactly matches the small asymmetry of the lagged correlations for the PDSI (the black line).

The above analysis was repeated for an MCDI time series derived for Northern California as described in appendix A. The results are very similar to those obtained for the Northern California PDSI. The lagged correlations between the Northern California MCDI and unsmoothed 500-hPa geopotential height anomalies shown by the dashed line in Fig. 1d are very similar to the lagged correlations for the PDSI. The differences are that the $e$-folding time of the correlations between the MCDI and 500-hPa height anomalies at negative lags is estimated to be slightly longer ( $\tau=6.3$ months compared with 6.1 months for the PDSI) and the correlation magnitudes are smaller at positive lags. The latter is expected since the MCDI does not depend on future precipitation (see, e.g., Fig. 11 of Part I).

The conclusion from this analysis of the N.CA PDSI and MCDI is that studies of atmospheric forcing of drought variability based on either of these drought indices should smooth the atmospheric forcing in a manner that is consistent with the smoothing that is implicit in the PDSI and MCDI. While this is perhaps obvious, choosing the appropriate smoothing requires determination of the exponential time scale of the PDSI and MCDI from a systematic procedure like that presented in appendix B of Part I. The previously noted MATLAB and Python codes that are available from the GitHub repository can be used for such purposes. Instructions for obtaining these codes along with three example applications are given in appendix A of Part I.

The PDSI and MCDI can alternatively be replaced with the hybrid precipitation index developed in Part I with the exponential time scale explicitly specified by the user to investigate precipitation variability on whatever time scale is of interest. In that case, the atmospheric forcing should be smoothed in the same manner as the hybrid precipitation index. This approach is used in the next section to investigate the nature of precipitation variability along the west coast of North America and its relationship to atmospheric forcing on time scales ranging from monthly to interannual, rather than being restricted to the geographically varying single time scale of the PDSI or MCDI for each region.

\section{Precipitation variability along the west coast of North America}

The algorithm for the hybrid precipitation index developed in Part I is applied in this section to investigate the dependence of geographical patterns of west coast precipitation variability on time scale and their relationships to atmospheric forcing. The analysis illustrates two advantages of the hybrid index over the PDSI and MCDI. First, time series of precipitation variability similar to the PDSI and MCDI are constructed as onesided, exponentially weighted averages of monthly precipitation anomalies for two regions for which PDSIs and MCDIs are not available. Second, the analysis is not restricted to the single time scale that is captured by the PDSI or MCDI; one-sided, exponentially weighted smoothing is applied consistently to the monthly precipitation anomalies in six regions along the west coast of North America using a wide range of exponential smoothing time scales.

Three of the six regions considered in this section are the Washington (WA), Oregon (OR), and N.CA regions that were analyzed in detail in Part I, and in section 2 of this study for the case of N.CA. Time series of monthly precipitation anomalies were similarly computed for Southern California (S.CA) and Southeast Alaska (S.AK) as described in appendix A. To fill the large geographical gap between S.AK and WA, we also constructed a regional precipitation time series for British Columbia (BC) from coastal station data (see appendix A).

As in the case of the nine regions in the lower 48 states analyzed in section 3 of Part I, PDSIs are available for 
NOAA climate divisions 6 and 7 to compute an estimated PDSI for S.CA as described in appendix A. An MCDI time series could likewise be computed for S.CA by a procedure analogous to that summarized in appendix A to obtain the N.CA MCDI time series for the analysis in section 2. PDSIs and MCDIs are not available for S.AK or BC. To investigate precipitation variability along the full west coast of North America, precipitation indices with exponentially decaying dependence on present and past precipitation analogous to the exponentially fading memories of the PDSI and MCDI were computed for all six regions based on the hybrid precipitation index developed in Part I that consists of one-sided exponentially weighted averages of present and past precipitation.

The ability to control the time scale of the exponential smoothing in the hybrid precipitation index is exploited in this section to investigate precipitation variability on time scales ranging from monthly to interannual by considering eight choices of $e$-folding time ranging from $\tau=0$ (corresponding to unsmoothed monthly precipitation anomalies) to 36 months that are consistently applied for each of the six regions. For the calculations in this section, the exponential weightings for all eight choices of $\tau$ were truncated at a lag of $K=100$ months (following the notation used in section 3 of Part I). This is approximately 3 times longer than the longest exponential smoothing time scale of $\tau=36$ months considered here.

It can be noted that the analysis in sections $3 \mathrm{~b}$ and $3 \mathrm{c}$ below that is based on the hybrid index could have been obtained from the PDSI or MCDI, but only for the regionally dependent single $e$-folding time that is implicit in each PDSI or MCDI time series, and only for the four of the six regions considered here for which PDSI and MCDI time series are available (WA, OR, N.CA, and S.CA). The dependencies of precipitation variability on time scale that are presented in sections $3 \mathrm{~b}$ and $3 \mathrm{c}$ could not be determined from the PDSI or MCDI. Furthermore, even if PDSI or MCDI time series were available for $\mathrm{S} . \mathrm{AK}$ and $\mathrm{BC}$, the regionto-region variation in the $e$-folding time scale that is implicit in the PDSI and MCDI would complicate the interpretation of the empirical orthogonal functions (EOFs) in section 3c. For example, the $e$-folding times of the exponential smoothing that is implicit in the PDSI time series for WA, OR, and N.CA are 3.6, 4.3, and 6.1 months, respectively (see Fig. 10 of Part I). An EOF analysis of PDSI or MCDI time series would partition the variance in unpredictable ways between the sought-after physical patterns of precipitation variability and the "noise" variance from the existence of higher-frequency variability in regions for which the implicit $e$-folding time scale of the PDSI or MCDI time series is short.

\section{a. Seasonal precipitation variability}

The seasonal cycles of the monthly averages and medians of precipitation and the standard deviations of the precipitation anomalies (defined to be the deviations of the monthly averages from the seasonal cycle) are shown by calendar month in Fig. 2 for the six west coast regions. In all six regions, the seasonal cycles of the standard deviations vary approximately in phase with the seasonal cycles of the monthly averages and medians. In OR, N.CA, and S.CA, the averages, medians and standard deviations are highest in the winter and decrease to near zero in the summer. The summertime averages, medians and standard deviations are slightly higher in WA than in the three more southerly regions. The much higher precipitation amounts and variability in S.AK and BC than in the other four regions are also notable, as is the fact that their maxima occur 1-3 months earlier than in the other four regions.

A characteristic of the precipitation variability that is important in the interpretation of the SPI analysis in section 4 and appendix $\mathrm{B}$ is the ratio of the range $\Delta \sigma$ of the 12 monthly standard deviations of the monthly precipitation anomalies to the average $\bar{\sigma}$ of the 12 monthly standard deviations. As indicated by the labels in the lower left corner of each panel of Fig. 2, this ratio is small in S.AK and BC and increases systematically southward from WA to S.CA. Consistent with the analysis of the SPI in section 2 of Part I, it is shown in appendix B of this study that larger values of $\Delta \sigma / \bar{\sigma}$ complicate the interpretation of the SPI for precipitation variability on time scales shorter than about 10 months.

\section{b. Regional relationships between precipitation and atmospheric variability}

Maps of the correlations between one-sided, exponentially weighted averages of precipitation anomalies (PCPexp) and concurrent and equivalently smoothed 500-hPa height anomalies (HGT500exp) over the northeast Pacific Ocean are shown based on year-round statistics in the upper group of panels in Fig. 3 for each of the six west coast regions and the eight choices of exponential time scale $\tau$ considered here. The geographical patterns of the correlations for each individual region are essentially the same, regardless of the degree of smoothing. This is evidence that the proximate causes of drought and precipitation variability in each region are independent of the time scale of the variability. For each region, smoothed precipitation variability is associated with a nearby localized area of negative 

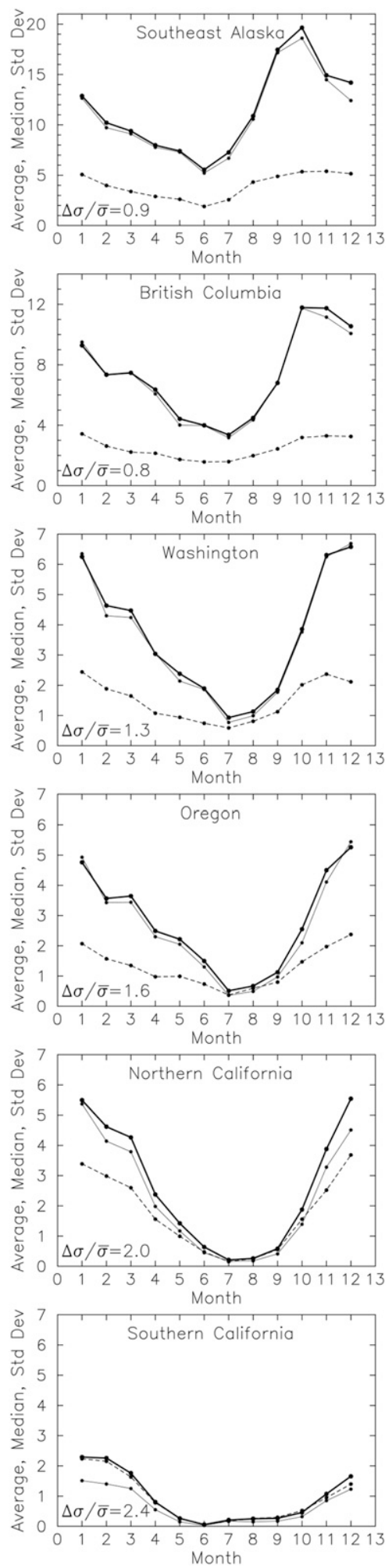

FIG. 2. The seasonal cycles of the average and median (thick and thin solid lines, respectively) and the standard deviation (dashed lines) of precipitation anomalies by calendar month during the period January 1948 through December 2016 for the six west coast regions considered in this study: Southeast Alaska (S.AK), British correlation with smoothed 500-hPa height anomalies to the northwest centered at the latitudes and longitudes listed in the caption for Fig. 4 below. The correlations of precipitation anomalies with atmospheric pressure anomalies to the northwest have been noted previously by Castello and Shelton (2004) for three of the regions considered here (WA, N.CA, and S.CA). Except for S.AK, the correlation maps in Fig. 3 show that local atmospheric forcing analogous to the wellknown ridging of the westerly atmospheric flow that has been studied extensively with regard to California drought variability (see section 2) is the dominant factor determining precipitation variability in every region from $\mathrm{BC}$ to S.CA.

S.AK is unique among the six regions in that the precipitation anomalies are also associated with a localized area of high positive correlation centered to the southeast over northern Washington and southwestern Canada (see Fig. 3a). An interpretation of this dipole of opposing correlations is given below. Other noteworthy features of the correlation maps in Fig. 3 are that the magnitude of the extremum negative correlation decreases with increased smoothing for S.AK (Fig. 3a), and to lesser extents BC and WA (Figs. 3b,c), but increases somewhat with increased smoothing for S.CA (Fig. 3f). This is evidence that precipitation variability with long time scales is less prevalent in the northern regions and most prevalent in S.CA. The extrema negative correlations are essentially independent of the time scale of the smoothing for OR and N.CA.

It was shown in section $3 \mathrm{a}$ that precipitation variability is highly seasonal in all six of the regions considered in this study and that there is almost no summertime precipitation in S.CA, N.CA, and OR, and very little summertime precipitation in WA (see Fig. 2). In consideration of this strong seasonality, the correlations between the one-sided exponentially weighted averages of precipitation and the 500-hPa height anomalies at the locations of maximum correlation in the upper group of panels in Fig. 3 were computed for each of the four 3-month seasons individually. The results are shown in Fig. 4 for exponential time scales of $\tau=0,3,6,12$, and 18 months; the correlations for

Columbia (BC), Washington (WA), Oregon (OR), Northern California (N.CA), and Southern California (S.CA). The S.AK, BC, N.CA, and S.CA regions were defined as summarized in appendix A. The WA and OR regions are statewide averages. Note the doubling and tripling of the dynamic ranges of the ordinates for BC and S.AK, respectively, compared with the other four regions. The fractional range $\Delta \sigma / \bar{\sigma}$ (see text) of the standard deviations that provides insight into the analysis of the SPI in appendix B is labeled at the bottom-left corner of each panel. 

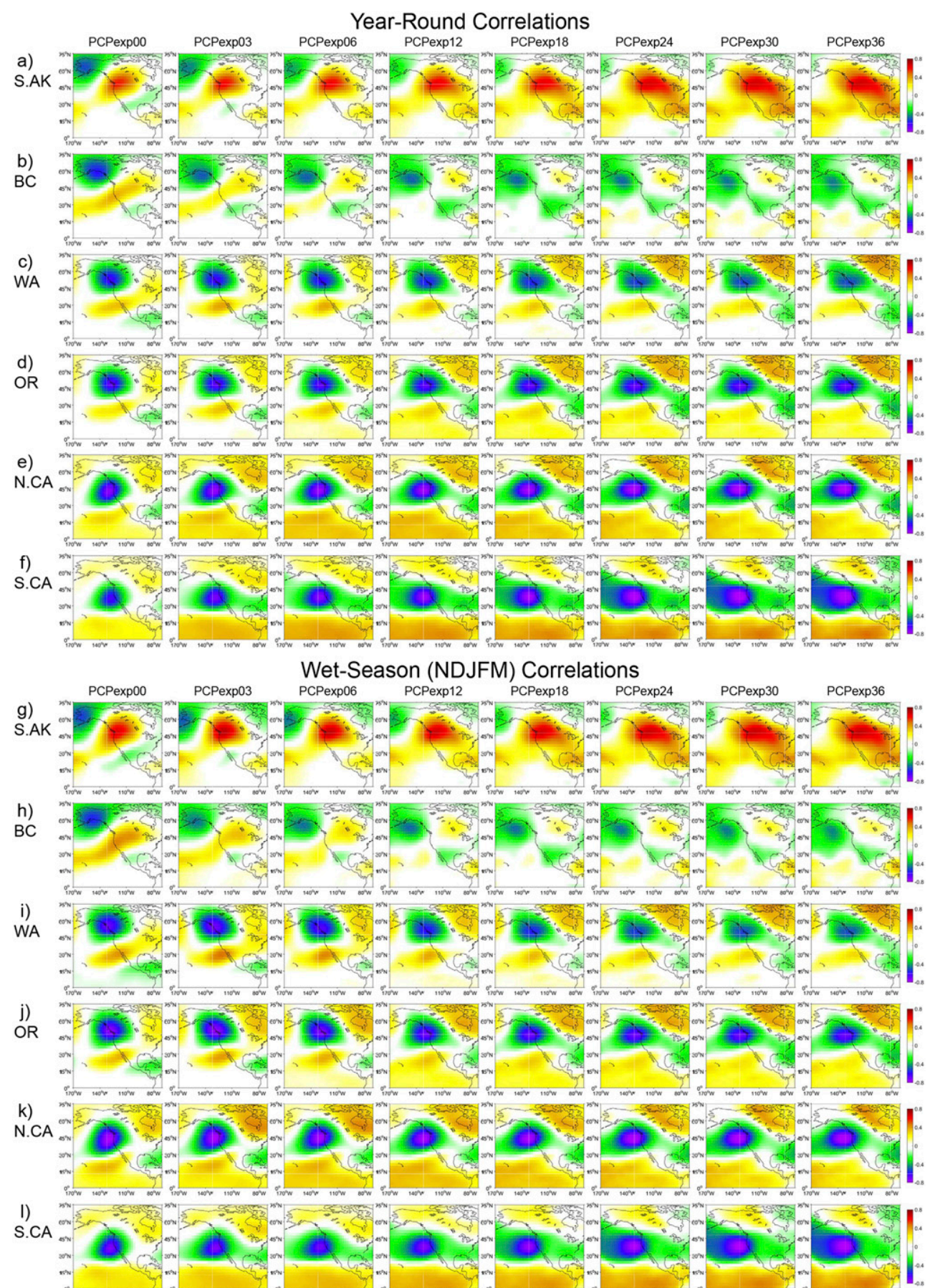

$\mathrm{m})$

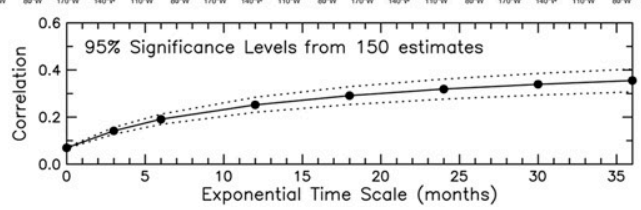

FIG. 3. Maps of the correlations between time series of one-sided, exponentially weighted averages of 500 -hPa geopotential height anomalies (HGT500exp) on a $2.5^{\circ} \times 2.5^{\circ}$ grid and equivalently smoothed precipitation anomalies (PCPexp) for each of the six regions along the west coast of North America considered in this study and the eight $e$-folding times of $\tau=0$ (unsmoothed), 3, 6, 12, 18, 24, 30, and 36 months 
$\tau>18$ months (not shown here) are very similar to those for $\tau=18$ months, except with slightly higher and lower correlation magnitudes south and north of OR, respectively.

It is evident from Fig. 4 that the correlations for winter, spring and fall are all similar to the year-round correlations (the black line in each panel) on all exponential time scales $\tau$, becoming increasingly similar with increasing $\tau$. The correlations for summertime data, however, are much weaker than for the other three seasons in the unsmoothed precipitation anomalies $(\tau=0)$ for the four southern regions. This is attributable to the previously noted weak summertime precipitation in these regions. The summertime correlation magnitudes improve rapidly with increased smoothing time scale $\tau$ in these four regions because the time span of the one-sided exponentially weighted averages includes progressively more effects of spring and winter precipitation with increasing $\tau$. For $\tau>$ 6 months, the summertime correlations in Fig. 4 become barely distinguishable from the year-round correlations or the correlations for the other three seasons.

The conclusion from Fig. 4 is that the year-round correlations in the upper group of panels of Fig. 3 are applicable to winter, spring, and fall for all exponential time scales $\tau$ but are applicable to summer only for $\tau>$ 6 months. To simplify the discussion that follows, we will consider only wet-season data hereafter, where we define the wet season to be the 5-month period NovemberMarch. This range of months was chosen taking into consideration the fact that the seasonal cycles of precipitation in S.AK and BC occur earlier than in the other four regions (see Fig. 2) and the fact that one-sided, exponentially weighted averages with nonzero $\tau$ include contributions from prior precipitation. Defined in this manner, the wet season accounts for $45 \%, 53 \%, 65 \%, 66 \%, 76 \%$, and $80 \%$ of the annual total precipitation amounts for S.AK, BC, WA, OR, N.CA, and S.CA, respectively.

While the analysis that follows is discussed in terms of wet-season variability, we note again that Fig. 4 shows that the results pertain to winter, spring, and fall on all time scales, as well as to summer on time scales of $\tau>6$ months.

Maps of the wet-season correlations between onesided, exponentially weighted averages of precipitation anomalies and concurrent and equivalently smoothed
500-hPa height anomalies are shown in the lower group of panels in Fig. 3. These maps are very similar to the maps of the correlations in the upper group of panels that were computed from year-round data. Close inspection reveals that the locations of the $2.5^{\circ} \times 2.5^{\circ}$ grid points of the 500-hPa height anomalies with maximum correlations for the wet-season data shift by modest amounts in three of the locations. In particular, the location of maximum correlation shifts westward by two grid points (about $320 \mathrm{~km}$ ) for BC and northwestward by one grid point in each dimension (about $340 \mathrm{~km}$ diagonally) for OR and N.CA. These geographical shifts are small in comparison with the breadths of the areas of high correlation for each region. The latitudes and longitudes of maximum wet-season correlation are listed in the caption for Fig. 5.

The wet-season statistics of the smoothed precipitation anomalies in each of the six west coast regions and the equivalently smoothed $500-\mathrm{hPa}$ height anomalies at the grid location in the center of the area of extremum negative correlation to the northwest of each region are summarized in Fig. 5. The wet-season standard deviations of smoothed precipitation anomalies are shown in Fig. 5a for all six regions and all eight choices of one-sided, exponentially weighted smoothing considered here. The standard deviations of unsmoothed monthly precipitation anomalies (the solid black line in Fig. 5a) vary latitudinally similarly to the latitudinal variations of the average wet-season total precipitation amounts shown by the black dashed line. Compared with the other regions, the wet-season standard deviations in N.CA and S.CA are disproportionately high compared with their average wet-season totals. The variability of the wet-season precipitation anomalies is much higher in S.AK than in any of the other five regions. The variability is about the same in BC and N.CA, both of which are higher than the variabilities in WA and OR. The variability is smallest in S.CA, but only slightly smaller than in WA and OR.

In all six regions, the wet-season standard deviations of precipitation anomalies decrease quite dramatically when smoothed with an $e$-folding time of only 3 months. Perhaps not surprisingly, precipitation variability everywhere along the west coast of North America is thus dominated by short-term variations that are greatly

(columns, from left to right). Correlations based on year-round statistics are shown for (a) S.AK, (b) BC, (c) WA, (d) OR, (e) N.CA, and (f) S.CA. (g)-(l) Correlation maps based only on wet-season statistics (November-March) are shown for the same six regions as (a)-(f). (m) The average and \pm 1 standard deviation of estimates of the $95 \%$ significance levels of the magnitudes of the correlations as functions of the $e$-folding time $\tau$ computed from correlations between each of the six pairs of PCPexp and HGT500exp time series at 25 grid locations on a $20^{\circ}$ longitude $\times 20^{\circ}$ latitude grid over the map domain (i.e., a total of $6 \times 25=$ 150 estimates). 

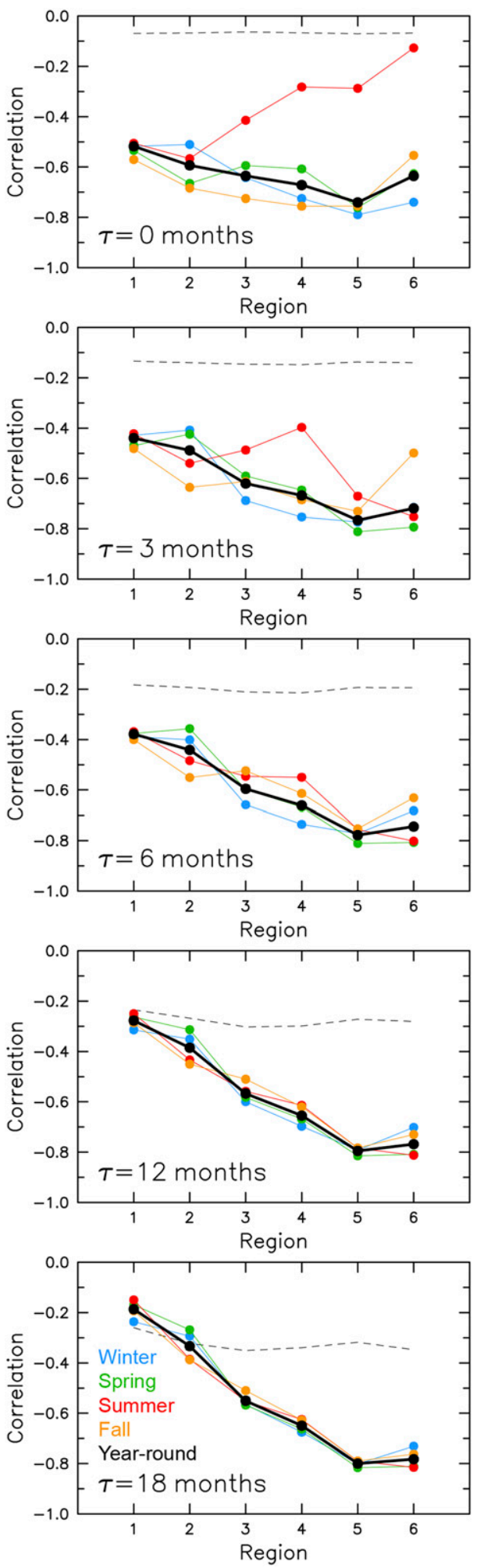

FIG. 4. The correlations between precipitation and 500-hPa geopotential height anomalies at the grid locations of maximum correlations in the upper group of panels in Fig. 3 for one-sided, exponentially weighted averages with $e$-folding times of $\tau=0$ (unsmoothed), 3, 6, 12, and 18 months (from top to bottom). attenuated with only a small amount of smoothing. The wet-season standard deviations continue to decrease gradually with further increases in the smoothing. The differences between the standard deviations of precipitation anomalies smoothed with $e$-folding times longer than 18 months are barely detectable.

The wet-season standard deviations of 500-hPa geopotential height anomalies (Fig. 5b) are similarly much smaller after smoothing with an $e$-folding time of only 3 months and continue to decrease gradually with further increases in the smoothing. Atmospheric forcing is thus also dominated by short-term variations along the entire west coast. The systematic northward increase of the 500-hPa height variability is presumably because of the closer proximity to the low pressure systems in the Gulf of Alaska that determine most of the variations in the largescale atmospheric pressure fields during the wet season.

The magnitudes of the negative wet-season correlations between concurrent smoothed precipitation and $500-\mathrm{hPa}$ height anomalies at the centers of the areas of extremum negative correlation (Fig. 5c) are largest in N.CA, slightly smaller in S.CA and decrease systematically northward from OR to S.AK. Local atmospheric forcing of precipitation variability by nearby pressure anomalies is thus most dominant in N.CA and S.CA and decreases in dominance toward the north. The decreases of the correlation magnitudes with increased smoothing for S.AK and the modest increase of the correlation magnitudes with increased smoothing for S.CA that were discussed qualitatively from Fig. 3 are quantified in Fig. 5c. The decreasing correlation magnitudes with increased smoothing in BC, WA, and OR in Fig. 5c show that the decreasing prevalence of precipitation variability with long time scales that was easy to see from Fig. 3 for S.AK also occurs in BC, WA, and OR, albeit to much lesser degrees. An interesting conclusion from Fig. $5 \mathrm{c}$ is that the correlation of N.CA precipitation variability with atmospheric forcing is essentially independent of time scale.

The six regions from S.AK to S.CA are numbered consecutively from north to south along the abscissa of each panel and the grid locations for the $500-\mathrm{hPa}$ height anomalies are 1) $62.5^{\circ} \mathrm{N}, 162.5^{\circ} \mathrm{W}$ for S.AK; 2) $55.0^{\circ} \mathrm{N}, 142.5^{\circ} \mathrm{W}$ for BC; 3) $52.5^{\circ} \mathrm{N}, 127.5^{\circ} \mathrm{W}$ for $\left.\mathrm{WA} ; 4\right)$ $47.5^{\circ} \mathrm{N}, 127.5^{\circ} \mathrm{W}$ for OR, 5) $42.5^{\circ}, 127.5^{\circ} \mathrm{W}$ for N.CA; and 6) $37.5^{\circ} \mathrm{N}$, $130.0^{\circ} \mathrm{W}$ for S.CA. The black solid line in each panel corresponds to the correlations based on year-round statistics. The colored lines according to the legend in the bottom panel are the correlations based on winter (December-February), spring (March-May), summer (June-August), and fall (September-November). The dashed line in each panel shows the estimated $95 \%$ significance levels of the correlations. 
a)

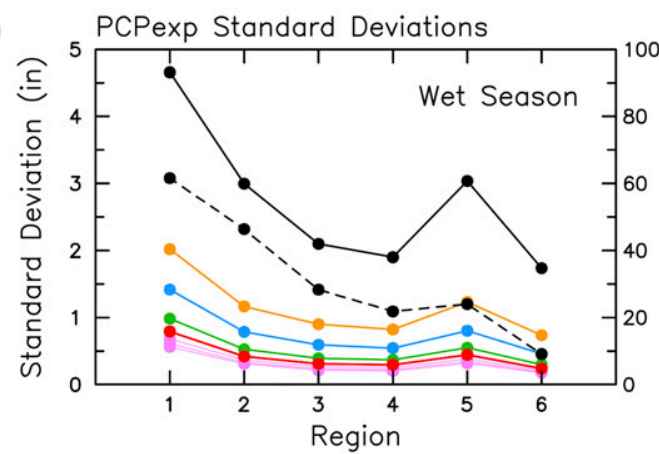

b)

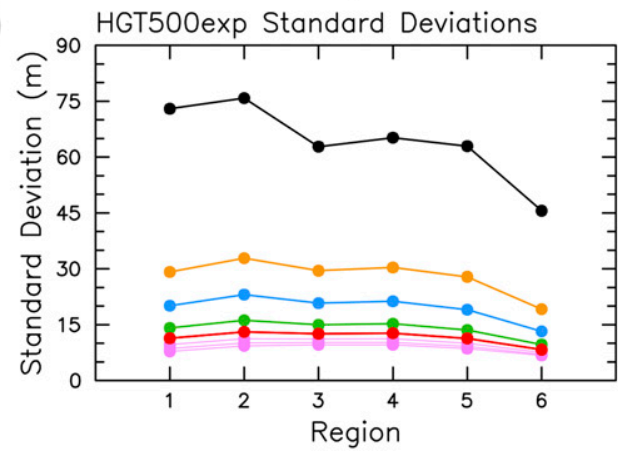

C)

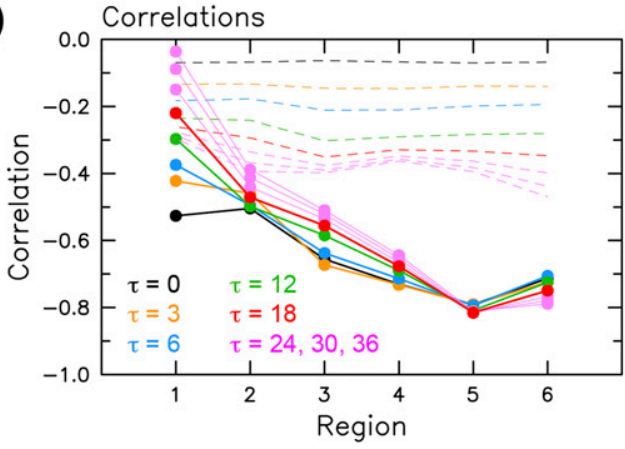

FIG. 5. Wet-season statistics for precipitation and 500-hPa geopotential height anomalies along the west coast of North America for eight choices of one-sided, exponentially weighted averaging: (a) The standard deviations of wet-season precipitation anomalies (solid lines) and the average wet-season total precipitation amounts as labeled on the right axis (dashed line); (b) the standard deviations of wet-season 500 -hPa height anomalies at the grid locations with extremum negative correlations in the lower group of panels in Fig. 3; and (c) the wet-season correlations between precipitation in each region and the $500-\mathrm{hPa}$ height anomalies at the same grid locations as in (b). The six regions from S.AK to S.CA are numbered consecutively from north to south along the abscissa of each panel and the grid locations for the 500-hPa height anomalies in (b) and (c) are 1) $62.5^{\circ} \mathrm{N}, 162.5^{\circ} \mathrm{W}$ for S.AK; 2) $55.0^{\circ} \mathrm{N}$, $147.5^{\circ} \mathrm{W}$ for $\left.\mathrm{BC} ; 3\right) 52.5^{\circ} \mathrm{N}, 127.5^{\circ} \mathrm{W}$ for $\mathrm{WA}$; 4$) 50.0^{\circ} \mathrm{N}, 130.0^{\circ} \mathrm{W}$ for OR, 5) $45.0^{\circ}, 130.0^{\circ} \mathrm{W}$ for N.CA; and 6) $37.5^{\circ} \mathrm{N}, 130.0^{\circ} \mathrm{W}$ for S.CA. The eight solid lines in each panel are color coded by the $e$-folding time of the exponential weighting according to the legend in (c). The dashed lines in the bottom panel are the estimated $95 \%$ significance levels of the correlations, color coded the same as the correlations.

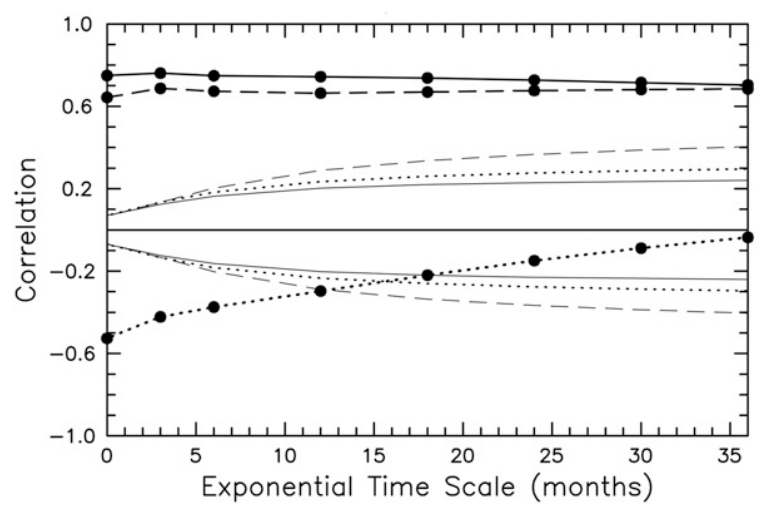

FIG. 6. The wet-season correlations between one-sided, exponentially weighted averages of S.AK precipitation and $500-\mathrm{hPa}$ geopotential height anomalies as functions of the $e$-folding time of the exponential weighting ranging from $\tau=0$ (unsmoothed) to 36 months. The dotted and dashed lines with the large dots correspond to the correlations with $500-\mathrm{hPa}$ height anomalies at, respectively, the northern $62.5^{\circ} \mathrm{N}, 162.5^{\circ} \mathrm{W}$ and southern $47.5^{\circ} \mathrm{N}, 120.0^{\circ} \mathrm{W}$ centers of extremum negative and positive correlations in Fig. $3 \mathrm{~g}$. The solid line with large dots corresponds to the correlations with the differences between the 500-hPa height anomalies at the southern location minus the northern location, which is interpreted here as an index for anomalies of the strength of the onshore flow aloft. The three pairs of thin lines without the large dots bracket the ranges within which correlations are statistically nonsignificant with $95 \%$ confidence for the correlations between S.AK precipitation and the northern (dotted lines) and southern (dashed lines) 500-hPa height anomalies and their differences (solid lines).

In addition to being negatively correlated with $500-\mathrm{hPa}$ height anomalies to the northwest, it was noted above from Fig. 3 that the precipitation variability in S.AK is positively correlated with 500 -hPa height anomalies to the southeast. The extremum of the region of positive correlation is located in northern Washington at $47.5^{\circ} \mathrm{N}$, $120.0^{\circ} \mathrm{W}$. The $500-\mathrm{hPa}$ height anomalies associated with the dipole of positive and negative correlations in all of the panels of Figs. $3 \mathrm{a}$ and $3 \mathrm{~g}$ correspond to stronger onshore geostrophic winds aloft during times of anomalously high precipitation and weakened onshore winds (negative anomalies) during times of anomalously low precipitation. The differences between the 500-hPa height anomalies at the two locations (the center of the positive correlation minus the center of the negative correlation) can be considered an index for the strength of onshore flow anomalies aloft.

The wet-season correlations between S.AK precipitation anomalies and 500-hPa height anomalies at the southern and northern centers of the dipole of positive and negative correlations are shown as a function of the $e$-folding time $\tau$ of the one-sided, exponential smoothing by, respectively, the dashed and dotted lines in Fig. 6. The dotted line shows the systematic decrease of the magnitude of the negative correlation with increased 


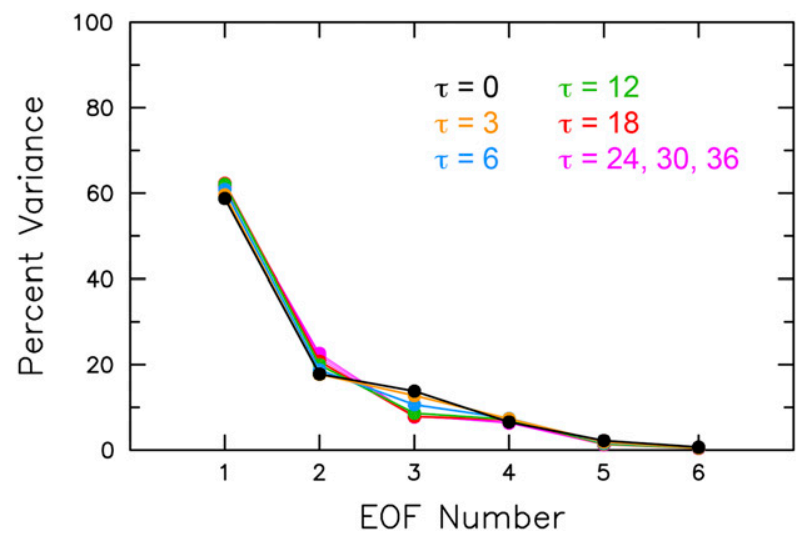

FIG. 7. The percentages of wet-season variance accounted for by each of the six EOFs of precipitation anomalies along the west coast of North America for eight choices of one-sided, exponentially weighted averaging. The eight lines are color coded by the $e$-folding time of the exponential weighting according to the legend.

smoothing time scale $\tau$ that was noted from the maps in Figs. 3a,g and 5c. These correlations become insignificant for $\tau \geq 18$ months, diminishing to zero for $\tau=$ 36 months and actually becoming slightly positive for larger $\tau$ (not shown here). In contrast, the positive correlations with 500-hPa height anomalies to the southeast (the dashed line) are larger in magnitude and essentially independent of the smoothing time scale. The wetseason correlations with the pressure difference index for the strength of the onshore flow anomalies (the solid line in Fig. 6) are even larger in magnitude with very little dependence on the smoothing time scale.

The distinction of the correlation patterns for S.AK in Fig. 3 compared with the correlation patterns in the other five regions, and the correlations in Fig. 6, can be interpreted as an indication that precipitation variability in S.AK is determined primarily by the strength of the onshore flow. This relation between S.AK precipitation variability and onshore flow has been noted previously by L'Heureux et al. (2004), Schnetzler and Dierking (2008), and Bieniek and Walsh (2017) and others. The analysis here shows that this relation is independent of the time scale of the precipitation variability.

We note that the onshore flow that causes increased precipitation in S.AK can be viewed as a northward deflection of the jet stream similar to the ridging from offshore high pressure anomalies that causes drought conditions in the regions to the south. This is discussed further from the EOF analysis in section 3c.

\section{c. Empirical orthogonal function analysis of precipitation variability}

The geographical patterns of covariability of precipitation anomalies along the west coast of North a)

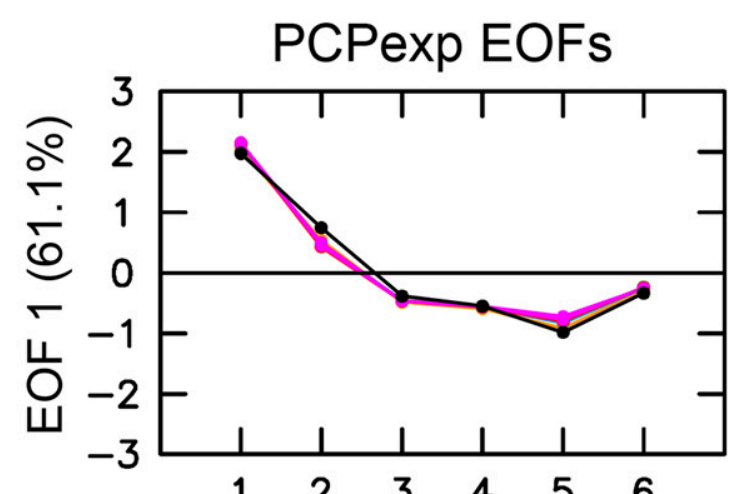

b)

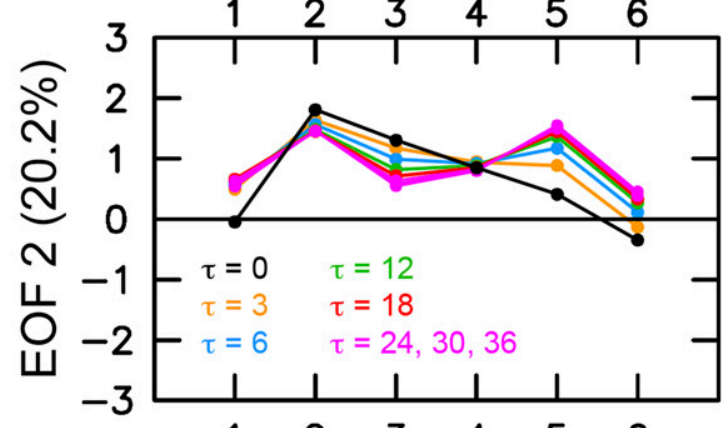

c)

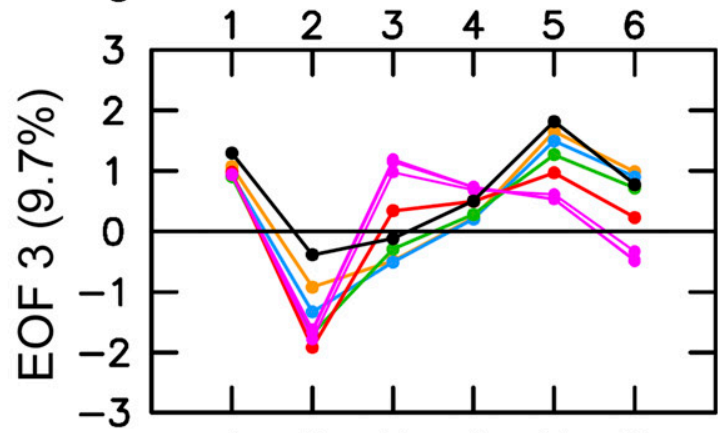

d)

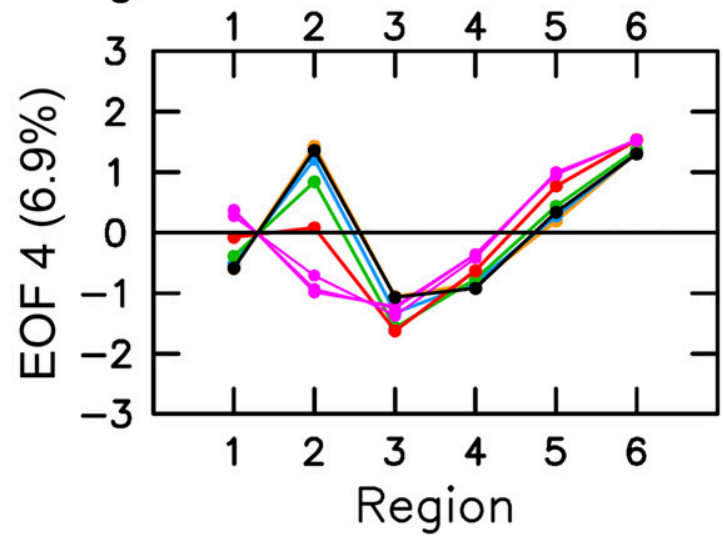

FIG. 8. (a)-(d) The first four EOFs computed from wet-season hybrid precipitation time series (PCPexp) along the west coast of North America for eight choices of one-sided, exponentially weighted averaging. The six regions from S.AK to S.CA are numbered consecutively from north to south along the abscissa of each panel and the eight lines in each panel are color coded by the $e$-folding time of the exponential weighting according to the legend in (b). The average percentage of variance accounted for by each EOF mode over the eight smoothing time scales $\tau$ is labeled on the ordinate of each panel. 
America can be characterized by EOF analysis (see, e.g., Hannachi et al. 2007). The percentages of total wetseason variance summed over the six regions that are explained by each of the six EOFs (Fig. 7) are nearly the same for every choice of the $e$-folding time $\tau$ of the smoothing considered here. Over the eight choices of $\tau$ ranging from 0 to 36 months, the averages of the percentages of wet-season variance explained by each of the four most dominant EOFs are $61.1 \%, 20.2 \%, 9.7 \%$, and $6.9 \%$, respectively, and the cumulative variance explained by these four leading EOFs exceeds $97 \%$ for every choice of $\tau$. The fifth and sixth EOFs thus account for very small amounts of the variance summed over the six regions and can therefore be considered noise in the sense that their patterns are determined mostly by the mathematical constraints of the orthogonality of the EOFs.

The first four EOFs of the smoothed wet-season precipitation anomalies in the six regions are overlaid in Fig. 8 for each of the eight choices of the $e$-folding time $\tau$ of the smoothing. These four modes are interpreted physically below. First, we summarize the salient features of the EOFs. The structure of the first EOF is nearly identical for every choice of $\tau$. The structures of the other three EOFs are more dependent on the smoothing time scale but are qualitatively very similar for each choice of $\tau$. The second EOF is most sensitive to $\tau$ in N.CA with weaker dependencies on $\tau$ in the other five regions. The third EOF is sensitive to $\tau$ in all regions except S.AK and OR. The fourth EOF is only sensitive to $\tau$ in $\mathrm{BC}$ with very little dependence on $\tau$ in the other five regions. A noteworthy feature is that the dependence on $\tau$ is weak in OR in all four EOFs. OR thus appears to be a "hinge point" in the covariability of precipitation anomalies elsewhere along the west coast that are associated with each of the EOF modes of variability. The different sensitivities of the second and third EOFs to $\tau$ are clues to the reason deduced below that these modes of variability are less robust over the eight choices of $\tau$ considered here.

While the sum of the variances in the six regions that is explained by a given EOF in Fig. 7 characterizes the overall importance of the EOF, this is often not a good measure of the importance of a particular EOF to the precipitation variability in any specific region. Nor is the magnitude of a particular EOF in a specific region necessarily an indication of the importance of that EOF to the variability in that region. This is especially the case for EOF applications such as this in which the precipitation time series for the different regions have a wide range of variances (see Figs. 2 and 5a). Physical interpretation of each EOF often becomes clearer from an examination of the fractions of the local variance accounted for in each region by each particular EOF. These fractions of local variance explained can be computed for each specific EOF as the squared correlation between the amplitude time series of the EOF and the smoothed time series of precipitation anomalies in each individual region.

The local percentages of variances explained in each region by the first EOF of wet-season precipitation variability (Fig. 9a) are essentially independent of the smoothing time scale $\tau$. For each of the eight choices of $\tau$, about $95 \%$ of the variance in S.AK is explained by the first EOF with substantially less of the variances explained in the other five regions. The first EOF can thus be interpreted as a "Southeast Alaska mode" of precipitation variability. Further support for this interpretation is provided by the maps in Fig. 10a that show the correlations between one-sided, exponentially weighted averages of concurrent wet-season 500-hPa height anomalies and the amplitude time series of the first EOF of wet-season precipitation variability for each of the eight smoothing time scales $\tau$. In all cases, the correlation maps are almost identical to the correlation maps between wet-season S.AK precipitation and 500-hPa height anomalies in Fig. 3g.

In association with this Southeast Alaska mode of wet-season precipitation variability, there are precipitation anomalies of the same sign but smaller magnitude in BC and of opposite sign and smaller magnitudes in the other four regions to the south (see Fig. 8a). Precipitation variability in S.CA is not well accounted for by the Southeast Alaska mode since less than $10 \%$ of the variance is explained for all choices of $\tau$ (see Fig. 9a). About $40 \%$ of the precipitation variances in OR and N.CA are explained on all time scales. In BC and WA, which straddle the node that separates the regions of positive and negative signs of the first EOF and are thus not as well characterized by this mode of variability, only $20 \%-30 \%$ of the variances are explained. The structure of the first EOF thus indicates that anomalously high precipitation in S.AK is associated mainly with anomalously low precipitation in OR and N.CA. Likewise, low precipitation in S.AK is associated with high precipitation in OR and N.CA. The magnitudes of the opposing anomalous precipitation amounts are nearly 4 times larger in S.AK than in OR and N.CA. While smaller in absolute magnitude in the southern regions, it is shown in appendix B that the precipitation anomalies in S.AK, OR, and N.CA are about the same in terms of the numbers of standard deviations from the norm in each region (see Figs. B2b,c).

The anticorrelation of OR and N.CA precipitation with S.AK precipitation can be explained by the proximity of the western portion of the region of positive 


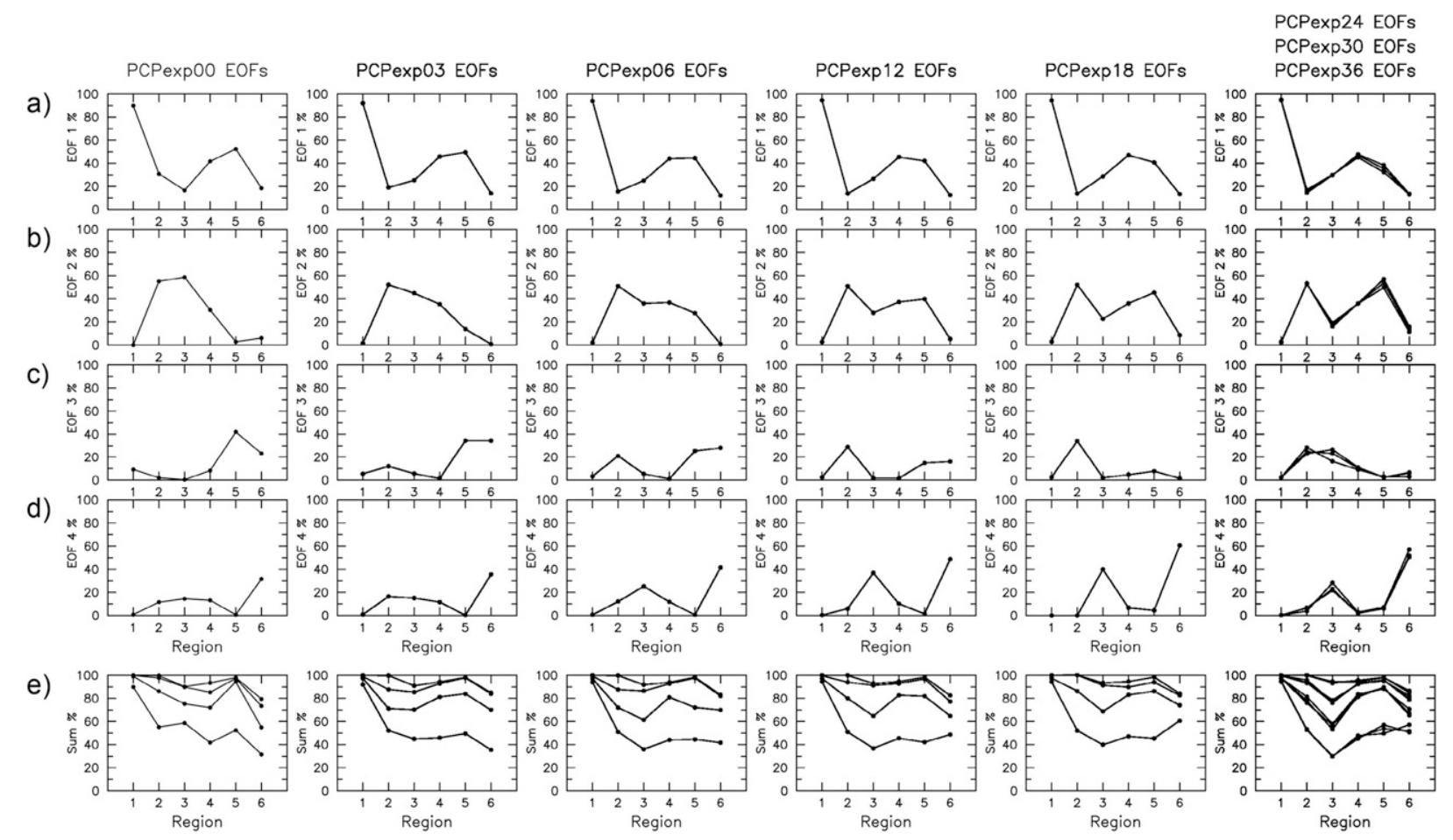

FIG. 9. (a)-(d) The local percentages of variance accounted for in each region by each of the first four EOFs computed from wet-season hybrid precipitation index time series (PCPexp) with eight choices of one-sided, exponentially weighted averaging. The six regions from S.AK to S.CA are numbered consecutively from north to south along the abscissa of each panel. The first five columns from left to right correspond to exponential weighting with $e$-folding times of $\tau=0$ (unsmoothed), 3, 6, 12, and 18 months, and the sixth column shows the superposition of the local percentages of variance accounted for with $e$-folding times of $\tau=24,30$, and 36 months. (e) The sums of the local percentages of variance accounted for in each region by optimal combinations of one, two, three, and all four (the lines in each panel, ordered from bottom to top) of the dominant four EOFs.

correlation in Fig. 10a to the locations of the extremum negative correlations of OR and N.CA precipitation with 500-hPa height anomalies off the coast of Oregon and Northern California (see Figs. 3j,k). The onshore flow that is associated with positive precipitation anomalies in S.AK can thus be interpreted as the northward deflection of the jet stream by the ridging that is associated with drought conditions in N.CA and OR. The above-noted smaller percentage of $\mathrm{BC}$ variance explained suggests that this onshore flow during dry spells in N.CA and OR tends to pass north of BC and therefore has less impact on the precipitation anomalies in $\mathrm{BC}$ than in S.AK.

Interpretations of the second and third EOFs of wetseason precipitation variability are more nuanced. The sensitivities of their spatial patterns to the smoothing time scale $\tau$ were noted above from Figs. 8b and 8c. The local variances explained are also dependent on $\tau$ (Figs. 9b,c). In the unsmoothed monthly anomalies $(\tau=0)$, the second EOF accounts primarily for the variances in $\mathrm{BC}$ and $\mathrm{WA}$ and to some extent $\mathrm{OR}$ but very little of the variances in the other three regions.
On the other hand, the third EOF of unsmoothed monthly anomalies accounts primarily for the variance in N.CA and S.CA. With increasing $\tau$, the second and third EOFs account, respectively, for progressively more and less of the N.CA variance.

The correlation maps in Figs. $10 \mathrm{~b}$ and 10c provide an interpretation of the above dependencies of the second and third EOFs on $\tau$. On short time scales, the extremum of the negative correlations between the amplitude time series of the second EOF and 500-hPa height anomalies in Fig. $10 \mathrm{~b}$ is in the same region where the $\mathrm{BC}$ precipitation anomalies are most strongly correlated with $500-\mathrm{hPa}$ height anomalies (see Fig. 3h). With increased smoothing time scale $\tau$, the center of the area of extremum negative correlation for the second EOF moves progressively southward, translating a distance of $850 \mathrm{~km}$ from $57.5^{\circ} \mathrm{N}, 150.0^{\circ} \mathrm{W}$ for $\tau=0$ to $50.0^{\circ} \mathrm{N}$, $147.5^{\circ} \mathrm{W}$ for $\tau=36$ months. Essentially the same southward shift with increasing $\tau$ occurs in the maps of the correlations of $\mathrm{BC}$ precipitation anomalies with $500-\mathrm{hPa}$ height anomalies in Figs. $3 \mathrm{~b}$ and $3 \mathrm{~h}$. This southward geographical shift with increased smoothing 


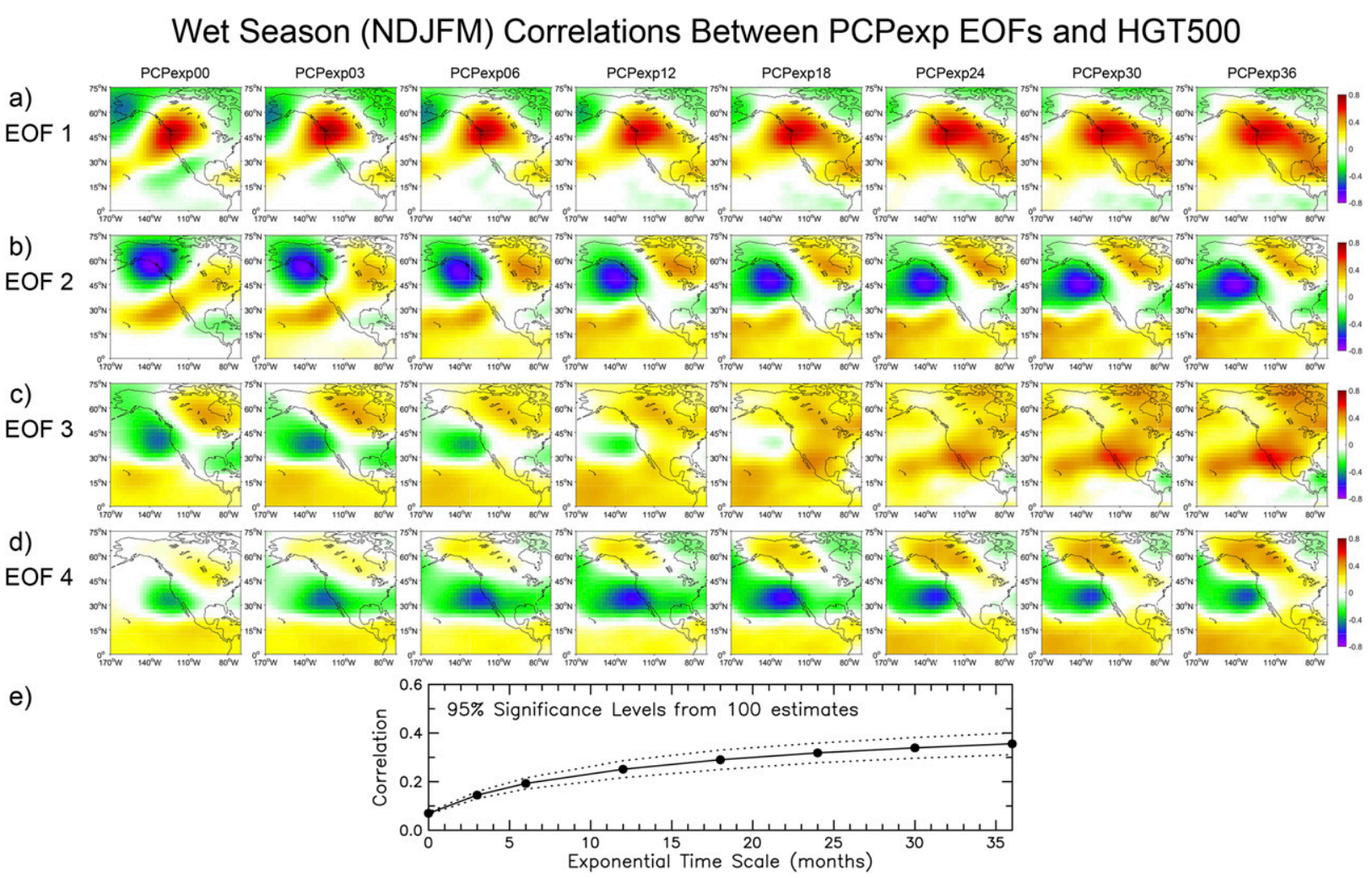

FIG. 10. (a)-(d) Maps of the correlations between time series of 500-hPa geopotential height anomalies on a $2.5^{\circ} \times 2.5^{\circ}$ grid and the amplitude time series of each of the first four EOFs of wet-season hybrid precipitation index time series (PCPexp) with eight choices of one-sided, exponentially weighted averaging. The eight columns from left to right correspond to the results for exponential weighting with $e$-folding times of $\tau=0$ (unsmoothed), $3,6,12,18,24,30$, and 36 months. (e) The average and \pm 1 standard deviation of estimates of the $95 \%$ significance levels of the magnitudes of the correlations as functions of the $e$-folding time $\tau$ computed from correlations between each of the four EOF amplitude time series and $500-\mathrm{hPa}$ height anomalies at 25 grid locations on a $20^{\circ}$ longitude $\times 20^{\circ}$ latitude grid over the map domain (i.e., a total of $4 \times 25=100$ estimates).

time scale $\tau$ exerts progressively greater influence on the ridging and troughing of the flow aloft that are associated, respectively, with drought and wet conditions in the more southerly regions, thus explaining the timescale dependencies of the local variances explained by the second EOF of precipitation variability.

Analogously, the dominance of the effects of the third EOF on the N.CA precipitation variability on short time scales is because of the proximity of the region of extremum negative correlation in Fig. 10c to the location of the $500-\mathrm{hPa}$ height anomalies off the coast of Northern California and southern Oregon that cause the ridging associated with drought variability in N.CA (see section 2; see also Figs. $3 \mathrm{j}$ and $3 \mathrm{k}$ ). The diminishing importance of the third EOF to the wet-season precipitation variability in N.CA with increasing $\tau$ is because of the diminishing magnitude of the negative correlations of the amplitude time series of the third EOF with smoothed 500-hPa height anomalies in Fig. 10c. In fact, the area of negative correlation in Fig. 10c becomes vanishingly small and is replaced with a region of positive correlation to the southeast for $\tau>18$ months. This decreasing correlation is accompanied by the above-noted increased correlation of the amplitude time series of the second EOF as the location of its strongest correlation with $500-\mathrm{hPa}$ height anomalies moves southward with increasing $\tau$.

The analysis above can be summarized succinctly as follows. On short time scales, the second and third EOFs of wet-season precipitation anomalies can be interpreted as, respectively, predominantly a localized "British Columbia mode" and predominantly a localized "California mode" of precipitation variability. With increasing time scale, these modes become progressively less distinct and are replaced with the very large-scale pattern of coherent precipitation variability in the second EOF with the same sign in all four regions from BC to N.CA (see Fig. 8b). This progression to a large-scale "West Coast mode" of synchronous precipitation variability of the same sign on the longer 
time scales exerts very little influence on the precipitation anomalies in the northernmost and southernmost regions considered here (S.AK and S.CA).

Physical interpretation of the fourth EOF of wetseason precipitation variability is somewhat risky since this mode accounts for only about $7 \%$ of the total variance summed over the six regions and is thus marginally distinct from the noise modes of the fifth and sixth EOFs. Moreover, the fourth EOF is mathematically constrained to be orthogonal to the three more energetic modes and therefore does not necessarily have a physical interpretation. Figures $9 \mathrm{~d}$ and $10 \mathrm{~d}$ nonetheless suggest that the fourth EOF can be interpreted as a "Southern California mode" of variability. One point of evidence in support of this interpretation is that the fourth EOF accounts for more of the wet-season precipitation variability in S.CA than in any of the other five regions on all time scales (Fig. 9d). Furthermore, the fourth EOF accounts for progressively more of the S.CA variability with increased smoothing time scale $\tau$, increasing from about $30 \%$ for the case of unsmoothed precipitation anomalies $(\tau=0)$ to more than $50 \%$ for smoothing time scales of $\tau \geq 12$ months.

Additional evidence that the fourth EOF is a Southern California mode of wet-season precipitation variability can be inferred from the correlation maps in Fig. 10d, which show that the amplitude time series of the fourth EOF for all smoothing time scales $\tau$ are negatively correlated with 500 -hPa height anomalies off central and Southern California in the same region of extremum negative correlation between S.CA precipitation anomalies and 500-hPa height anomalies in Fig. 31. The magnitudes of the negative correlations in Fig. 10d increase systematically with increased smoothing time scale up to $\tau=18$ months, synchronous with the increases in the local S.CA variance explained by the fourth EOF in Fig. 9d.

It is noteworthy that the fourth EOF of wet-season precipitation variability also accounts for progressively more of the WA precipitation variability with increased smoothing time scale, increasing from about $15 \%$ for $\tau=0$ to $40 \%$ for $\tau=18$ months, then decreasing somewhat for $\tau \geq 24$ months (see Fig. 9d). From the correlation maps in Fig. 10d, the increases in the magnitudes of the negative correlations with increasing $\tau$ are associated with increased positive correlations of the amplitude time series of the fourth EOF with $500-\mathrm{hPa}$ height anomalies in a region in northwestern Canada. This dipole of correlations with opposing signs is indicative of offshore geostrophic flow anomalies near $50^{\circ}$ latitude that are associated with this EOF on long time scales. The systematic increase of the local percentage of smoothed WA precipitation variance explained by the fourth EOF (Fig. 9d) and the negative sign of the fourth EOF in WA (Fig. 8d) are indicative of a positive correlation between onshore geostrophic flow anomalies and positive precipitation anomalies in WA in association with the ridging that decreases the precipitation in S.CA. This suggests that the moisture-laden storms that are deflected north during drought conditions in S.CA tend to come onshore in WA, i.e., farther south than the previously discussed onshore flow in S.AK that is associated with drought conditions in OR and N.CA. The connection between the opposite-signed S.CA and WA precipitation anomalies tends to become stronger with increased smoothing time scale $\tau$ (see Figs. 9d and 10d).

The fourth EOF of wet-season precipitation variability accounts for relatively little of the variances in N.CA and OR (Fig. 9d), which straddle the node that separates positive and negative signs of the EOF (Fig. 8d) and are thus not well characterized by this mode of variability. The small variances accounted for in S.AK and BC by the fourth EOF indicate that these regions are evidently too far north to be affected by the onshore and offshore flow anomalies associated with the opposite-signed S.CA and WA precipitation anomalies.

Because the EOF amplitude time series are uncorrelated, the local precipitation variance explained in each region by any linear combination of the EOFs is just the sum of the local percentages of variance explained by each individual EOF included in the linear combination. The percentages of wet-season variance explained by optimal combinations of one, two, three and all four of the four most dominant EOFs are shown in Fig. 9e. As discussed above, nearly all of the precipitation variance in S.AK is accounted for by just the first EOF on all time scales. On monthly time scales $(\tau=0)$, nearly $90 \%$ of the variance in $\mathrm{BC}$ is accounted for by the first and second EOFs. Similarly, more than $90 \%$ of the variance on short time scales in N.CA is accounted for by just two EOFs, but in this case the first and third EOFs. On longer time scales, the three most dominant EOFs are required to account for $90 \%$ or more of the variances in both $\mathrm{BC}$ and N.CA. The causes of precipitation variability in BC and N.CA thus become progressively more multifaceted with increasing time scale of the variability.

The nature of wet-season precipitation variability in the other three regions (WA, OR, and S.CA) is multifaceted on all time scales. At least three of the dominant EOFs are required to account for more than $90 \%$ of the variances in WA and OR on all time scales. West coast precipitation variability is most complex in S.CA where the variance is smallest (Fig. 5a). As discussed above, the variance in S.CA is primarily explained by the fourth 
EOF, but it accounts for only $30 \%$ of the variance of monthly anomalies $(\tau=0)$, increasing to $60 \%$ of the variance for $\tau=18$ months. Depending on $\tau$, the four dominant EOFs account for $80 \%-85 \%$ of the S.CA variability. The fifth EOF must be included to account for $90 \%$ or more of the variance of S.CA precipitation anomalies.

\section{West coast precipitation variability from the standardized precipitation index}

The primary motivations for developing the hybrid precipitation index in Part I were to enable the construction of precipitation time series with the exponentially decaying dependence on present and past precipitation that is implicit in the PDSI and MCDI, with the added ability to characterize precipitation variability on any time scale of interest by specifying the $e$-folding time of the exponential weighting. The ability to control the time scale of the smoothing with the hybrid precipitation index is a feature that is shared in common with the SPI. A question that naturally arises is whether the analysis and conclusions of this study could have been achieved from the SPI. Aside from the fact that the block-average dependence of the SPI on present and past precipitation is less physical than the exponentially fading memory that the hybrid index shares in common with the PDSI and MCDI, the answer to this question is nuanced.

For the investigation of wet-season precipitation variability on a region-by-region basis in section $3 b$, it is shown in appendix B (see Fig. B1c) that use of SPIxx time series smoothed with accumulation time scales $\mathrm{xx}$ chosen to be consistent with the $e$-folding times $\tau$ of the hybrid index considered in this study would reach essentially the same conclusions as those obtained in section $3 b$ from the hybrid index for the wet season November-March. Note, however, that a rigorous analysis of atmospheric forcing of precipitation variability from SPIxx time series analogous to the investigation of atmospheric forcing carried out in section $3 b$ from the hybrid index should be based on time series of $500-\mathrm{hPa}$ geopotential height on the $2.5^{\circ} \times 2.5^{\circ}$ grid smoothed in a manner consistent with the complicated details of the SPI algorithm.

We also reiterate the important point from section 2 of Part I that analysis of precipitation variability from SPIxx time series for regions like WA, OR, N.CA, and S.CA where precipitation amounts are always small during the summertime (see Fig. 2) would be compromised if summertime data were included in the analysis in section 3 (see Figs. 4-7 of Part I). This is because the normalization procedure of the SPI algorithm scales the accumulated precipitation anomalies by a different standard deviation for each of the 12 calendar months. It is apparent from Figs. B1a and B1b in appendix B and the discussion in section 2 of Part I that the effects of seasonality of the standard deviation on the SPIxx time series are an especially complicating issue for accumulation time scales xx shorter than about 10 months, becoming less so with increasing $\mathrm{xx}$.

The details of the normalization procedure in the SPI algorithm that complicate the interpretation of the SPI in any individual region also lead to additional complications in the interpretation of patterns of precipitation covariability determined from SPIxx time series in the six regions that were considered in the EOF analysis of the time series of the hybrid precipitation index in section 3c. As discussed in appendix B (see also section 2 of Part I), this issue arises from the fact that all information about actual precipitation amounts is removed by the normalization. The numerical value of an SPIxx time series for any particular month and any specific region is effectively the number of standard deviations by which the precipitation differs from the median value for the associated calendar month and region. Since the standard deviation used to scale the accumulated precipitation values in the SPI algorithm differs for each of the 12 calendar months, as well as for each region, a given numerical value of an SPIxx time series corresponds to a larger precipitation amount in a month or region with large standard deviation than in a month or region with small standard deviation. The complicating effects of the annual variation of the standard deviation in each region decrease with increasing accumulation time scale $\mathrm{xx}$ because of the diminishing annual variation of the standard deviation with increased smoothing (see the colored lines in the middle panels in Fig. 5 of Part I).

Irrespective of the complications introduced by annual variations of the standard deviations, which become relatively minor as noted above for accumulation time scales xx longer than about 10 months, the covariability of SPIxx time series addresses a different question from that considered in this study. Specifically, the EOF analysis in section $3 \mathrm{c}$ identified patterns of covariability of anomalous precipitation amounts on monthly to interannual time scales. In contrast, an EOF analysis of SPIxx time series (see Fig. B2b) identifies patterns of covariability of the numbers of standard deviations of the precipitation anomalies from the regionby-region norms. The geographical patterns of the EOF modes of covariability of this unitless nonphysical variable differ in general from the EOF patterns of the precipitation anomalies themselves (see Figs. B2a,b). A notable exception is that the first EOF computed from 
the two precipitation indices has similar geographical structure, a point that is returned to below.

In addition to the complexity of the scaling of accumulated precipitation values by a different standard deviation for each of the 12 calendar months, each region, and each choice of smoothing time scale $\mathrm{xx}$, the SPI algorithm includes a nonlinear transformation of the distribution of precipitation over the historical data record for each of the 12 calendar months. It is thus not possible to invert the SPI procedure to reconstruct the accumulated anomalous precipitation amounts exactly from the SPIxx time series to investigate the patterns of covariability of precipitation anomalies identified in section $3 \mathrm{c}$.

While the SPI is not well suited to studies of the covariability of anomalous precipitation amounts for the reasons summarized above, use of SPIxx time series to investigate covariability of precipitation anomalies in terms of the approximate numbers of standard deviations from the norms is a valid and useful characterization of precipitation variability that may be of interest in some studies. Indeed, it is shown in appendix B that the first EOF of wet-season precipitation anomalies computed from SPIxx time series provides insight into the interpretation of the first EOF computed in section $3 \mathrm{c}$ from the hybrid precipitation index. Specifically, the smaller precipitation amounts in WA, OR, N.CA, and S.CA that are associated with precipitation anomalies of opposite sign in S.AK in the dominant mode of wetseason precipitation variability (Fig. 8a and the top panel of Fig. B2a) turn out to be approximately equal in terms of the numbers of standard deviations from the individual norm in each region (the top panel of Fig. B2b). It is also shown in appendix B that essentially equivalent insight can be obtained by simply normalizing each hybrid precipitation index time series by its standard deviation (the top panel of Fig. B2c), thus avoiding the computational and interpretational complexities of the SPI algorithm.

\section{Discussion and conclusions}

The hybrid precipitation index developed in Part I was applied in this study to investigate the nature of precipitation variability along the west coast of North America from Southeast Alaska to Southern California. We first examined the relationship between atmospheric forcing and the Palmer drought severity index (PDSI) and the model calibrated drought index (MCDI) for N.CA in section 2. The correlations of both the PDSI and the MCDI with unsmoothed monthly 500-hPa geopotential height anomalies underestimate the effects of atmospheric forcing on precipitation because unsmoothed $500-\mathrm{hPa}$ height variability is inconsistent with the filtering that is implicit in the PDSI and MCDI (Figs. 1c,d). The filtering properties of the PDSI and MCDI were quantified in section 3 of Part I with the help of the hybrid precipitation index. The N.CA PDSI and MCDI are much more highly correlated with $500-\mathrm{hPa}$ height anomalies filtered in a manner that is consistent with the filtering in the PDSI or MCDI (Figs. 1e,f).

The analysis in section 2 thus concludes that the N.CA PDSI and MCDI are essentially responses to the integrated effects of present and past atmospheric forcing with a fading memory that depends on the one-sided, exponential weighting that is implicit in the PDSI and MCDI. (The PDSI also depends weakly on future atmospheric forcing, see section 2.) Since the intrinsic time scales determined by the PDSI and MCDI algorithms vary regionally, and in general differ from each other for a given region (see section 3 of Part I), the analysis procedure in appendix B of Part I must be carried out region by region to determine the appropriate exponential weighting time scale to apply to atmospheric data in order to investigate atmospheric forcing of precipitation variability as characterized by the PDSI or MCDI for any particular region. The MATLAB and Python codes that can be obtained from the GitHub repository as explained in appendix A of Part I can be used for such purposes.

A recognized limitation of the PDSI is that it is not well suited to studies of the relationships between drought variations in different regions because of the region-by-region differences between the intrinsic time scale determined by the PDSI algorithm (Heim 2002; Karl 1983; Guttman 1992, 1998; Wells et al. 2004; Wu and Kinter 2009; Williams et al. 2017). For example, the $e$-folding times of the exponential smoothing that is implicit in the PDSI time series for WA, OR, and N.CA are 3.6, 4.3, and 6.1 months, respectively (see Fig. 10 of Part I). The same limitation applies to the MCDI. Moreover, neither the PDSI nor the MCDI can be used to investigate drought variations on multiple time scales. Furthermore, the PDSI and MCDI are only available for the contiguous lower 48 states of the United States.

To these ends, the hybrid precipitation index developed in Part I is useful for deriving PDSI/MCDI-like precipitation indices for any desired time scale using a straightforward procedure that consists of computing one-sided, exponentially weighted averages of present and past precipitation with a specified $e$-folding time. The above-noted MATLAB and Python codes can be used to compute the hybrid precipitation index from any monthly averaged time series of precipitation anomalies. The ability to specify the time scale of interest is similar to that of the standardized precipitation index 
(SPI), but without the interpretational complications because of the details of the normalization procedure in the SPI algorithm (see section 4 and appendix B; see also the discussion in section 2 of Part I). Moreover, the uniform weighting of past precipitation in the SPI is less physical than the exponential weighting that the hybrid index shares in common with the PDSI and MCDI.

The hybrid precipitation index was applied in section 3 to investigate precipitation variability along the west coast of North America on monthly to interannual time scales from one-sided, exponentially weighted averages with $e$-folding times $\tau$ ranging from 0 (corresponding to unsmoothed monthly anomalies) to 36 months. For context, we determined empirically that the time span of one-sided, uniformly weighted averages that are most highly correlated with one-sided, exponentially weighted averages with an $e$-folding time of $\tau$ is approximately $1.25 \tau$, which in practice must be rounded to the nearest integer value. It is shown in section 2 of Part I that such one-sided, uniformly weighted averages are essentially equivalent to the SPIxx with the same time span $\mathrm{xx}$, especially for $\mathrm{xx}$ longer than about 10 months (i.e., $\tau$ longer than about 8 months). The longest exponential smoothing time scale of $\tau=36$ months considered in this study is similar to an SPI smoothing time span of about 45 months.

The analysis in section 3 focused on precipitation variability during the wet season, defined herein to be the 5-month period November-March. It was shown, however, that the conclusions of this study pertain to winter, spring and fall on all time scales, as well as to summer for smoothing with $e$-folding times $\tau$ longer than 6 months (see Fig. 4).

The proximate cause of wet-season precipitation variability in each of the west coast regions except S.AK is the same for all of the time scales of variability considered in this study (see Fig. 3). The smoothed precipitation variability is negatively correlated with smoothed 500-hPa height anomalies centered in a nearby area to the northwest of each region. These correlation maps are evidence that local atmospheric forcing analogous to the well-known ridging of the westerly atmospheric flow that affects California drought variability is the dominant factor determining the precipitation variability in every region from BC to S.CA. This has been shown previously by Castello and Shelton (2004) for three of the regions considered in this study (WA, N.CA, and S.CA). For time scales of 3 months and longer, the latitudinal variation of the correlation magnitudes (Fig. 5c) indicates that this local atmospheric forcing is most dominant in N.CA and S.CA with systematically decreasing dominance with increasing latitude to the north of N.CA.
The cause of precipitation variability in S.AK is distinctly different from the other west coast regions. The correlation patterns in Figs. 3a and 3g and the correlations with the index of onshore flow aloft in Fig. 6 indicate that S.AK precipitation on all of the time scales considered here is controlled primarily by the strength of the onshore flow in Southeast Alaska. This relationship between S.AK precipitation anomalies and onshore flow anomalies is captured as the dominant mode of west coast wet-season precipitation variability in Fig. 8a. Maps of the wet-season correlations between 500-hPa height anomalies and the amplitude time series of the first EOF (Fig. 10a) are almost identical to the maps of the correlations between $500-\mathrm{hPa}$ height anomalies and the S.AK precipitation time series in Fig. $3 \mathrm{~g}$. This "Southeast Alaska mode" of precipitation variability accounts for about $95 \%$ of the S.AK precipitation variance for all smoothing time scales $\tau$.

The association of S.AK precipitation with southwesterly onshore flow aloft has been noted previously by L'Heureux et al. (2004), Schnetzler and Dierking (2008), and Bieniek and Walsh (2017) and others. The highest precipitation anomalies generally occur during warm and wet winters. The onshore advection of moisture is associated with atmospheric pressure anomalies with the same dipole structure as the dipole patterns of correlation in the maps in Figs. 3a,g and 10a. The analysis in this study shows that S.AK precipitation variability is controlled by onshore flow on all time scales ranging from monthly to interannual.

The dominant EOF of west coast wet-season precipitation variability in Fig. 8a also reveals that increased precipitation in S.AK is associated with decreased precipitation from WA to S.CA, accounting for about $40 \%$ of the variances in OR and N.CA. This dominant mode of west coast precipitation variability thus indicates that the positive onshore flow anomalies that cause increased S.AK precipitation are correlated with a northward deflection of the jet stream similar to that associated with the ridging that causes drought conditions from WA to S.CA. The northward deflection evidently mostly passes to the north of $\mathrm{BC}$ since the first EOF explains relatively little of the precipitation variability in BC. Although the precipitation amounts from WA to S.CA are much smaller than the precipitation amounts of opposite sign in S.AK in this dominant mode of variability, it is shown in appendix B that the precipitation anomalies in terms of the numbers of standard deviations from the norm in each region are approximately equal in S.AK, WA, OR, and N.CA and slightly smaller in S.CA.

The geographical patterns of wet-season precipitation variability elsewhere along the west coast of North America are more complex than the precipitation 
variability in S.AK. Some salient characteristics can nonetheless be inferred from the EOF analysis in section 3c. On short time scales, the second and third EOFs can be interpreted as a "British Columbia mode" and a "California mode," respectively. Because the amplitude time series of EOFs are uncorrelated, this indicates a weak correlation between the precipitation variability in $\mathrm{BC}$ and N.CA on these short time scales. With increasing time scale, the third EOF diminishes in importance and the structure of the second EOF evolves into a pattern that represents a "West Coast mode" of synchronous precipitation anomalies of the same sign from BC to N.CA, accounting for $30 \%-40 \%$ of the variances in these regions but essentially none of the variances in S.AK and S.CA.

Precipitation variability in S.CA is the most complex of the six west coast regions considered in this study. The fourth EOF, referred to here as a "Southern California mode," explains less than half of the wet-season variance in S.CA. The remainder is distributed between the other EOFs, and the fifth EOF must be included to account cumulatively for $90 \%$ or more of the variance. The structure of the fourth EOF indicates that a substantial portion of wet-season precipitation variability in WA varies out of phase with S.CA precipitation variability, especially on longer time scales. This out-of-phase relationship can be taken as evidence that the moistureladen storms that are deflected northward by ridging during persistent drought conditions in S.CA tend to increase the precipitation in WA from increased onshore flow. They have little effect on the precipitation in OR and N.CA, which straddle the node that separates positive and negative signs of the fourth EOF.

The analysis in this study illustrates the utility of the hybrid precipitation index developed in Part I. The hybrid index enabled determination of the time scales of the N.CA PDSI and MCDI, which provided insight in section 2 into the most suitable choices of temporal smoothing for the investigation of atmospheric forcing of California drought variability on the single time scales that are captured by the PDSI or MCDI algorithms. The simplicity of the algorithm for the hybrid index facilitated the construction in section 3 of PDSI/MCDI-like precipitation indices for the $\mathrm{S}$. $\mathrm{AK}$ and $\mathrm{BC}$ regions for which actual PDSI and MCDI time series are not available. The ability to control the time scale of interest with the hybrid index enabled the investigation in section 3 of the dependencies of the geographical patterns of precipitation variability on time scale. This is an important advantage of the hybrid index since precipitation variability occurs on a wide range of time scales, not on just the single time scale identified by the PDSI or MCDI algorithms.
Acknowledgments. This research was partly supported by NASA Contract NNH15CO77C to Remote Sensing Systems. We thank Roger Samelson and Nicholas Siler for their suggestions for improving the manuscript. We also thank two anonymous reviewers for helpful comments that improved the manuscript.

\section{APPENDIX A}

\section{Data Sources}

The time series of monthly precipitation anomalies for N.CA that is analyzed in section 2 was defined to be the average of the monthly precipitation anomalies over NOAA climate divisions 1, 2, 3, and 5 in California (see Fig. A1). The precipitation time series for the individual climate divisions were downloaded for the time period January 1948-May 2017 from the NOAA Climate at a Glance web page (http://www.ncdc.noaa.gov/cag/). A Palmer drought severity index (PDSI) does not appear to be available for N.CA. We therefore estimated the N.CA PDSI as the average of the individual PDSIs downloaded from the above NOAA Climate at a Glance web page for California climate divisions 1,2, 3, and 5 . Because of the nonlinearity of the iterative algorithm for the PDSI, the N.CA PDSI calculated in this manner is not exactly the same as the N.CA PDSI that would be calculated properly from the area-weighted average precipitation and air temperature anomalies in the four climate divisions.

To assess the accuracy of our estimated N.CA PDSI, we compared properly calculated statewide PDSIs obtained from the above NOAA Climate at a Glance web page for CA, OR, and WA with estimates computed as the average of the PSDIs calculated properly by NOAA for each of the individual climate divisions in each state. For all three states, the correlations between the properly calculated and estimated statewide PDSI were 0.95 . We infer from this that our estimated PDSI for N.CA that is analyzed in section 2 is likely very similar to what would be obtained from a properly calculated N.CA PDSI.

A model calibrated drought index (MCDI) time series for N.CA was somewhat crudely defined for the analysis in section 2 by approximating NOAA climate divisions $1,2,3$, and 5 by the sum of the two rectangles in Fig. A1. The northern rectangle is the region $38.0^{\circ}-$ $42.0^{\circ} \mathrm{N}$ and $120.0^{\circ}-124.5^{\circ} \mathrm{W}$ and the southern rectangle is the region $34.5^{\circ}-38.0^{\circ} \mathrm{N}$ and $118.5^{\circ}-121.0^{\circ} \mathrm{W}$. Monthly time series of the MCDI on a $1 / 8^{\circ}$ grid were downloaded for the time period January 1948-May 2017 from https://www.ldeo.columbia.edu/ williams/ se_drought_2017_jgr.html. We used the "all_forcings" 


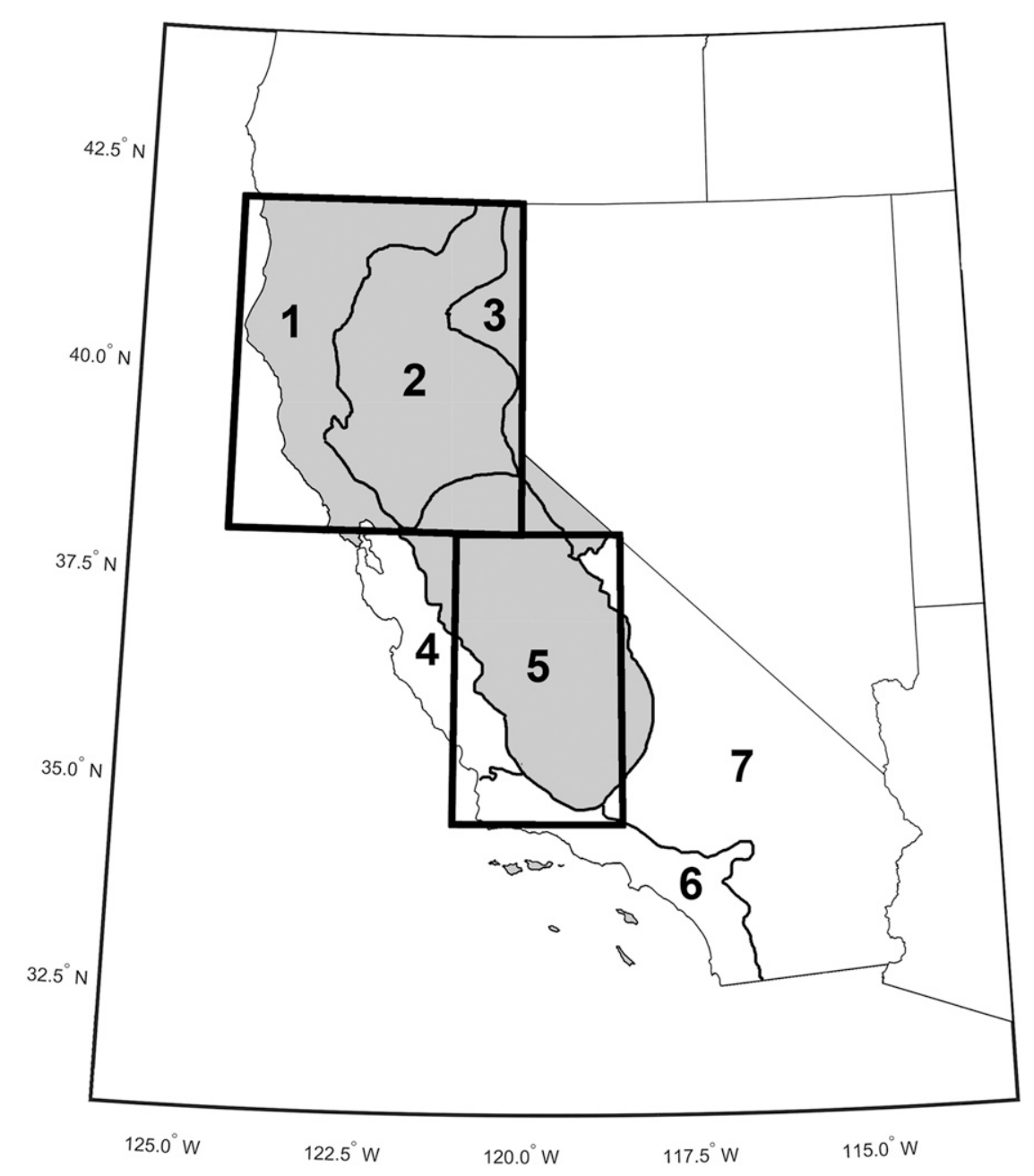

FIG. A1. A map showing the seven NOAA climate divisions for California. The N.CA region was defined for this study to be the area enclosed by climate divisions $1,2,3$, and 5. The S.CA region was defined to be the area enclosed by climate divisions 6 and 7 . The two rectangles indicate the region over which the monthly MCDI time series on a $1 / 8^{\circ}$ grid were averaged to define an approximate N.CA MCDI time series for the analysis in section 2.

version of the data file that was computed from meteorological variables obtained from phase 2 of the North American Land Data Assimilation System project (NLDAS2) (Xia et al. 2012a,b) for the time period 19792017 for which the NLDAS2 data are available. Prior to 1979 , this version of the MCDI time series was computed from various alternate climatological datasets (see the metadata in the NetCDF file). The MCDI time series for N.CA was derived by averaging the $1 / 8^{\circ}$ gridded MCDI time series over the two rectangular regions in Fig. A1.

The time series of monthly precipitation anomalies for S.AK, WA, OR, and S.CA that are analyzed in section 3 were downloaded for the time period January 1948-May 2017 from the above NOAA Climate at a Glance web page. The precipitation time series for WA and OR were defined to be statewide averages. The time series for
S.CA was defined to be the average of the monthly precipitation anomalies over NOAA climate divisions 6 and 7 in California (see Fig. A1). The central California coastal mountain range (NOAA climate division 4) was excluded from both the N.CA and S.CA regional time series because its characteristics differed distinctly from those of the other six regions. The S.AK time series was defined to be the average of monthly precipitation anomalies for the four NOAA climate divisions in southeast Alaska (the Northeast Gulf region, the North Panhandle region, the Central Panhandle region, and the South Panhandle region) where the highest precipitation rates occur in the state of Alaska (see, e.g., Fig. 1 in L'Heureux et al. 2004).

To fill the large geographical gap between S.AK and WA, monthly time series of precipitation for British 
Columbia were downloaded for the time period January 1948-December 2016 from http://climate.weather.gc.ca/ historical_data/search_historic_data_e.html for the nine coastal locations McInnes Island, Dryad Point, Bella Coola, Sandspit, Langara Island, Estevan Point, Cape Scott, Port Hardy, and Prince Rupert. The time period for this BC station precipitation data is 5 months shorter than the time period for the other precipitation data summarized above. The analysis in section 3 was therefore restricted to the January 1948-December 2016 time period of availability of precipitation data for BC. A regional monthly precipitation time series for $\mathrm{BC}$ was constructed as the arithmetic average of the monthly average station data at the above nine locations.

The monthly SPIxx time series of standardized precipitation indices that are analyzed in appendix B were computed from the above monthly precipitation data using the computer software downloaded from the University of Nebraska-Lincoln website https://drought.unl.edu/ droughtmonitoring/SPI/SPIProgram.aspx.

The monthly averages of 500-hPa geopotential height fields that are analyzed in sections 2 and 3 were obtained from the NOAA National Centers for Environmental Prediction (NCEP) Reanalysis V1 fields (Kalnay et al. 1996) that are produced on a $2.5^{\circ} \times 2.5^{\circ}$ grid and were downloaded from the web from https://www.esrl.noaa.gov/ psd/data/gridded/data.ncep.reanalysis.html.

\section{APPENDIX B}

\section{Comparisons of Hybrid and SPI Time Series of West Coast Precipitation Variability}

An advantage of the hybrid precipitation index over the PDSI and MCDI drought indices is the ability to investigate precipitation variability on any desired time scale by choosing the $e$-folding time $\tau$ of the one-sided, exponentially weighted average smoothing. With appropriate choice of $\tau$, a hybrid index can be derived that has the same exponentially fading memory of past precipitation as the PDSI or MCDI for any particular region (see Fig. 1 of this study and Figs. 10 and 11 in Part I). Unlike the hybrid index, however, the time scales of the PDSI and MCDI cannot be adjusted.

The adjustability of the time scale of the hybrid index is a feature that is shared in common with the standardized precipitation index (SPI) developed by McKee et al. (1993) and Edwards and McKee (1997) (see also the summary in section 2 of Part I). Besides the fact that the block-average dependence of the SPI on past precipitation is less physical as a drought index than the exponentially fading dependencies of the PDSI, MCDI, and hybrid indices, the complicated details of the SPI algorithm lead to interpretational issues that are discussed in detail with examples in section 2 of Part I. The purpose of this appendix is to assess the degree to which the analysis of wet-season precipitation variability from the hybrid precipitation index time series considered in sections $3 b$ and $3 c$ of this study could be done from SPI time series.

For the region-by-region analysis of wet-season precipitation variability in section $3 b$, we determined empirically that the block-average accumulation time span $\mathrm{xx}$ that gives the SPIxx time series that is most highly correlated with a hybrid index with an $e$-folding time of $\tau$ is approximately $\mathrm{xx}=1.25 \tau$. The correlations between the SPIxx time series with seven choices of $\mathrm{xx}$ and analogously smoothed time series of the hybrid index with $\tau=\mathrm{xx} / 1.25$ are shown in Fig. B1 for each of the six west coast regions considered in this study.

The correlations computed from year-round statistics (Fig. B1a) decrease systematically from north to south and increase systematically with increased smoothing. From the detailed analysis and interpretation of SPIxx time series in section 2 of Part I, it can be anticipated that the geographical variation of the correlations in Fig. B1a is attributable to the complicated normalization procedure of the SPI algorithm that scales the precipitation anomaly for a given month by a standard deviation that is computed separately from the historical record for each of the 12 calendar months. A consequence of this procedure is that correlations based on year-round statistics are lower in regions where there is large annual variation of the 12 monthly standard deviations (see Figs. 4 and 5 of Part I).

The degree to which the annual variation of the standard deviation of precipitation anomalies affects the time series computed by the SPI algorithm can be characterized by the fractional range $\Delta \sigma / \bar{\sigma}$ of the 12 monthly standard deviations that is labeled in the lower left corner of each panel of Fig. 2, where $\Delta \sigma$ is the annual range of the 12 monthly standard deviations and $\bar{\sigma}$ is the average value of the 12 monthly standard deviations. As shown by the dashed line in Fig. B1a, $\Delta \sigma / \bar{\sigma}$ increases from north to south in tandem with the north-to-south decrease of the year-round correlations between the SPIxx time series and analogously smoothed time series of the hybrid index that are shown by the solid lines in Fig. B1a.

The southward increase of $\Delta \sigma / \bar{\sigma}$ in Fig. B1a is associated with the fact that precipitation amounts are always small during the summertime at the more southerly locations (see Fig. 2). This summertime influence is confirmed from Figs. B1b and B1c that show the correlations based only on summer and wet-season data, respectively. The correlations from summer data show 
a)

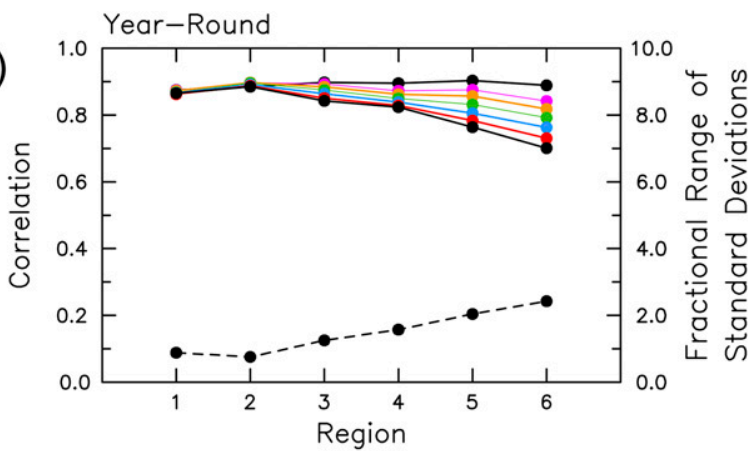

b)

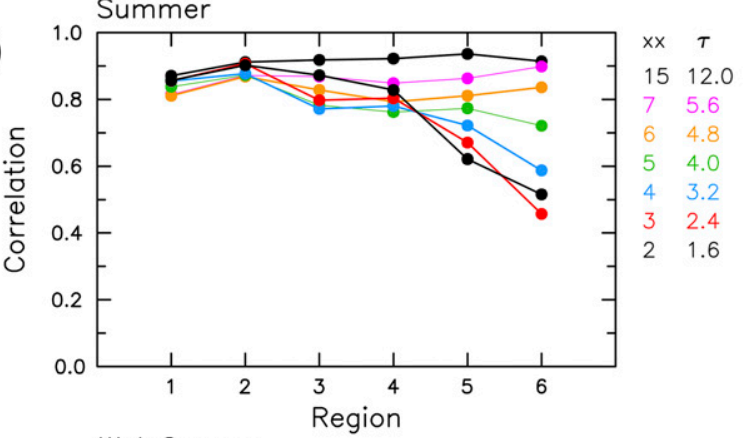

c)

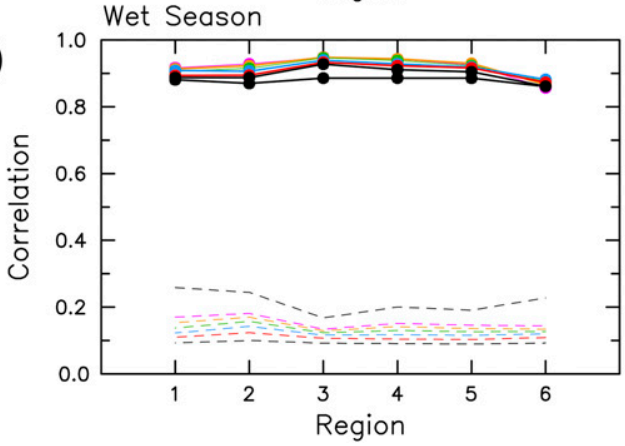

FIG. B1. The correlations of SPIxx time series for seven choices of block-average accumulation time scale xx with analogously smoothed time series of the hybrid precipitation index with $e$-folding times of $\tau=\mathrm{xx} / 1.25$. The six regions from S.AK to S.CA are numbered consecutively from north to south along the abscissa of each panel, and the seven lines in each panel are color coded by the smoothing time scale $\mathrm{xx}$ and associated $e$-folding time $\tau$ according to the legend to the right of (b). The correlations are shown based on statistics computed from (a) year-round data, (b) summer data (June-August), and (c) wet-season data (the 5-month period November-March). The dashed line in (a) is the fractional range of standard deviations $\Delta \sigma / \bar{\sigma}$ (see text). The dashed lines in (c) are the estimated $95 \%$ significance levels of the correlations, color coded the same as the correlations.

north-to-south decreases similar to those of the yearround correlations in Fig. B1a, except with smaller and somewhat noisier correlations. In contrast, there is very little geographical variability of the correlations from wet-season data.

The analysis in section 2 of Part I also explains why the correlations in Figs. B1a and B1b (and to a lesser extent,
Fig. B1c) increase with increased smoothing time scale $\mathrm{xx}$. The annual variation of the 12 monthly standard deviations used for the normalization by calendar month in the SPI algorithm decreases and converges toward a constant value with increased accumulation time span $\mathrm{xx}$ (see the colored lines in the middle panels of Fig. 5 of Part I). This is because the accumulated precipitation in summertime values of the SPIxx includes more and more spring and winter precipitation with progressively larger $\mathrm{xx}$. The effects of seasonality of the standard deviation become small for accumulation time scales $\mathrm{xx}$ longer than about 10 months.

The conclusion that can be drawn from Fig. B1c is that the region-by-region analysis of the wet-season precipitation variability in section $3 \mathrm{~b}$ could essentially have been done from SPIxx time series. This would not have been the case if the analysis in section $3 \mathrm{~b}$ had included summertime data, at least not on time scales $\mathrm{xx}$ shorter than about 10 months.

The question of whether SPIxx time series could have been used in section $3 c$ to identify patterns of covariability of wet-season precipitation variability over the six regions along the west coast of North America has a more complicated answer. The numerical value of the SPIxx time series for any particular month in a particular region can be interpreted as the approximate number of standard deviations by which the precipitation anomaly for that month differs from the median value for that calendar month over the historical data record for that region. This interpretation would be exact if the monthly precipitation values over the historical data record were Gaussian distributed for each of the 12 calendar months, thus obviating the need for the fitting and nonlinear transformation of the distribution of precipitation variability for each calendar month that is part of the SPI algorithm. As noted previously and discussed in more detail in section 2 of Part I, the scaling of precipitation values in the SPI algorithm removes all information about the actual accumulation of precipitation. Moreover, because the scaling is different for each of the 12 calendar months, the lost information about actual precipitation amounts differs for each calendar month. This is further complicated by the nonlinearity of the transformation of the distribution of precipitation over the historical record for each calendar month in the SPI algorithm.

The complexities of the interpretation of SPIxx time series summarized above are mitigated to some extent with increasing time scale $\mathrm{xx}$ of the smoothing because of the above-noted convergence of the 12 monthly standard deviations of precipitation anomalies toward a constant value with increasing $\mathrm{xx}$. For the west coast regions considered in this study, the complexities of the interpretation can also be mitigated for small values of $\mathrm{xx}$ by excluding summertime 
a) $\quad$ PCPexp $\tau=0,3,6,12,18,24$ mos
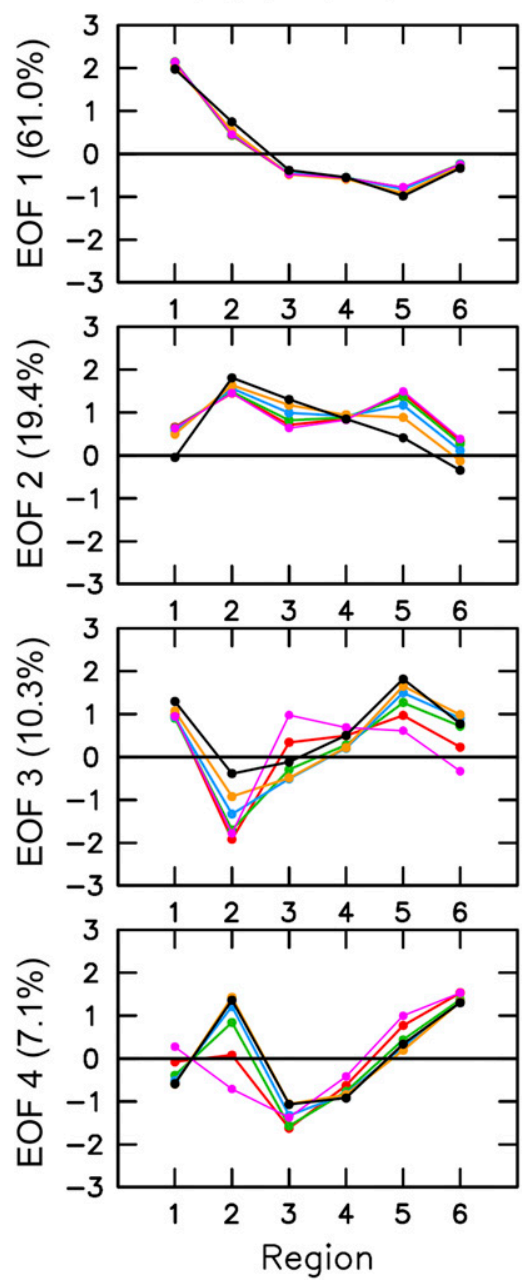

b) $x x=1,4,8,15,23,30$ mos
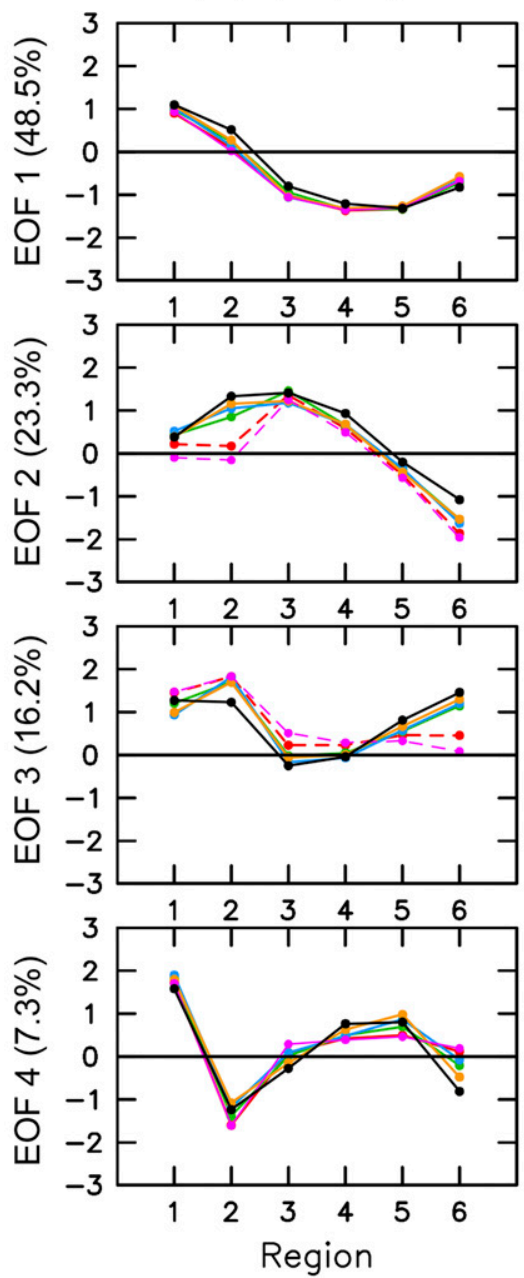

C) Standardized PCPexp $\tau=0,3,6,12,18,24 \mathrm{mos}$
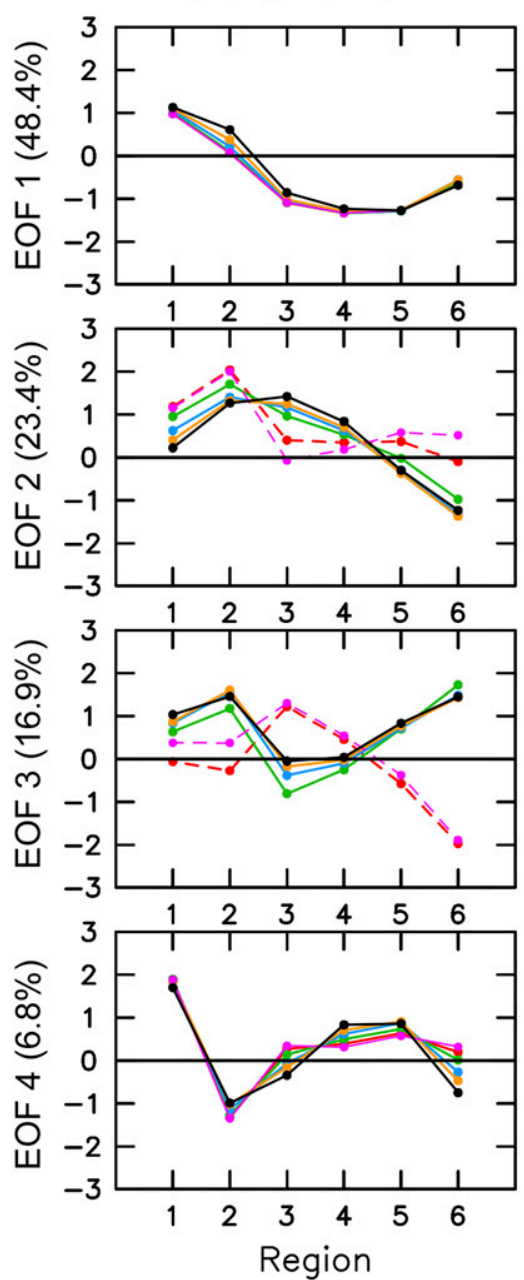

FIG. B2. The first four EOFs of wet-season precipitation anomalies along the west coast of North America from three different precipitation indices and six choices of smoothing. The six regions from S.AK to S.CA are numbered consecutively from north to south along the abscissa of each panel, and the six lines in each panel are color coded by the smoothing time scale labeled at the top of each column. The percentages of variance accounted for by each EOF mode are very nearly the same for each choice of smoothing time scale; the average values over the six smoothing time scales are labeled on the ordinate of each panel. The three precipitation indices are (a) the hybrid precipitation index PCPexp with $e$-folding times of $\tau=0,3,6,12,18$, and 24 months; (b) the SPIxx index with the nearest-integer values of block-average accumulation time scales of $\mathrm{xx}=1.25 \tau=1,4,8,15,23$, and 30 months; and (c) the standardized hybrid index with the same $e$-folding times $\tau$ as in (a). The EOFs shown by red and purple dashed lines in (b) and (c) draw attention to the switching of the order of standardized PCPexp EOFs 2 and 3 for smoothing time scales of $\tau=18$ and 24 months compared with the SPIxx EOFs for the equivalent accumulation time scales of $\mathrm{xx}=23$ and 30 months.

data, as in the wet-season analysis in section $3 \mathrm{c}$. The standard deviations still vary region by region. A given numerical value of an SPIxx time series thus corresponds to a larger precipitation amount in a region with large standard deviation than in a region with small standard deviation.

The above mitigation strategies do not eliminate the nonlinear transformation of the distribution of precipitation that is part of the SPI algorithm. It is therefore not possible to invert the SPI procedure to reconstruct the accumulated anomalous precipitation amounts exactly from an SPIxx time series to investigate the patterns of covariability of precipitation anomalies that were identified in section 3c. Analysis of precipitation variability from SPIxx time series is thus restricted to investigation of the patterns of covariability of the relative degrees to which the anomalous accumulated precipitation was unusual in each individual region compared with historical statistics for that region. This can be interpreted 
as the covariability of (approximately) the region-byregion numbers of standard deviations from the median values of the precipitation in the six regions.

The first four EOFs of smoothed wet-season SPIxx time series in the six regions along the west coast of North America are shown in Fig. B2b for six choices of the accumulation time scale $\mathrm{xx}=1.25 \tau$ that correspond to the hybrid index time series (PCPexp) analyzed in section 3 for the cases of the six $e$-folding times of $\tau=0,3,6,12,18$, and 24 months. For easy reference, the first four EOFs of the PCPexp time series from Fig. 8 for these six choices of $\tau$ are shown in Fig. B2a. For both sets of precipitation indices and all six matched choices of smoothing parameters $\tau$ and $\mathrm{xx}$, the geographical structures of the first EOF are similar with a zero crossing between BC and WA. The correlations between the amplitude time series of the first EOF computed from PCPexp and SPIxx time series are about 0.75 for each matched pair of smoothing parameters $\tau$ and $\mathrm{xx}$. There is no clear association between the geographical structures of the other three EOFs computed from the two sets of precipitation indices.

The similarity of the first EOF computed from the two sets of precipitation indices and the high correlation between their amplitude time series provide useful insight into the interpretation of this mode of variability. As discussed in section 3c, the first EOF computed from the PCPexp time series consists of out-of-phase variations north and south of the zero crossing between $\mathrm{BC}$ and WA. The positive onshore flow anomalies that cause above-normal precipitation in S.AK are thus associated with below-normal precipitation in WA, OR, N.CA, and S.CA. Likewise, anomalously low precipitation in S.AK is associated with anomalously high precipitation from WA to S.CA. The amplitudes of the precipitation anomalies associated with this mode of variability are much smaller in the region from WA to S.CA than in S.AK.

Although the magnitudes of the anomalous precipitation amounts associated with the dominant mode of variability are smaller from WA to S.CA, the structure of the first EOF computed from the SPIxx time series indicates that the precipitation amounts are equally anomalous in terms of the numbers of standard deviations from the norms in each region. In particular, when the precipitation in S.AK is one standard deviation above normal for that region, the precipitation in WA, OR, and N.CA is about one standard deviation below the normal amounts in each of those three regions and slightly less than one standard deviation below normal in S.CA.

This tandem interpretation of the dominant mode of variability in terms of wet-season precipitation amounts from the EOFs of PCPexp time series and the numbers of standard deviations from the norms from the EOFs of SPIxx time series can be equivalently achieved from the
PCPexp time series without the computational complexities of the SPIxx algorithm. This can be done by simply scaling the wet-season PCPexp time series for each of the six regions by the standard deviation computed region-by-region from the wet-season months of November-March over the full data record. The EOFs of the resulting standardized PCPexp time series are shown in Fig. B2c. For all six choices of smoothing, the geographical structures of the EOFs of standardized PCPexp are essentially the same as those of the EOFs of the SPIxx time series. One minor qualifier is that the ordering of the second and third EOFs computed from standardized PCPexp with smoothing time scales of $\tau=$ 18 and 24 months (shown by the dashed lines in Fig. B2c) is reversed from the ordering of the second and third EOFs computed from the SPIxx time series with the matched accumulation time scales of $\mathrm{xx}=23$ and 30 months (shown by the dashed lines in Fig. B2b).

We note that the correlations between the amplitude time series of the first EOFs of the PCPexp and standardized PCPexp time series are about 0.84 for each $e$-folding time $\tau$. The somewhat lower correlations of about 0.75 between the first EOFs of the PCPexp and SPIxx time series noted above are attributable to the complexities of the SPI algorithm.

For the analysis of west coast precipitation variability in this study, we conclude that the SPIxx precipitation indices offer no significant advantage in the interpretation of patterns of covariability of precipitation in the six regions considered here. If the interest is in determining geographical patterns of the relative degrees to which the anomalous precipitation was unusual in each individual region compared with historical statistics for that region, the computational and interpretational complexities of the SPI algorithm can be avoided by considering the standardized PCPexp hybrid precipitation index.

\section{REFERENCES}

Alley, W. M., 1984: The Palmer drought severity index: Limitations and assumptions. J. Climate Appl. Meteor., 23, 1100-1109, https://doi.org/10.1175/1520-0450(1984)023<1100:TPDSIL> 2.0.CO;2.

Beguería, S., S. M. Vicente-Serrano, F. Reig, and B. Latorre, 2014: Standardized precipitation-evapotranspiration index revisited: Parameter fitting, evapotranspiration models, tools, datasets and drought monitoring. Int. J. Climatol., 34, 3001-3023, https:// doi.org/10.1002/joc.3887.

Bieniek, P. A., and J. E. Walsh, 2017: Atmospheric circulation patterns associated with monthly and daily temperature and precipitation extremes in Alaska. Int. J. Climatol., 37, 208-217, https://doi.org/10.1002/joc.4994.

Castello, A. F., and M. L. Shelton, 2004: Winter precipitation on the U.S. Pacific coast and El Niño-Southern Oscillation events. Int. J. Climatol., 24, 481-497, https://doi.org/10.1002/joc.1011. 
Chelton, D. B., 1983: Effects of sampling errors in statistical estimation. Deep-Sea Res., 30A, 1083-1103, https://doi.org/10.1016/ 0198-0149(83)90062-6.

— by the SPI, PDSI, and MCDI. Part I: Development of the Index. J. Hydrometeor., 21, 1945-1976, https://doi.org/10.1175/ JHM-D-19-0230.1.

Davis, R. E., 1977: Techniques for statistical analysis and prediction of geophysical fluid systems. Geophys. Astrophys. Fluid Dyn., 8, 245-277, https://doi.org/10.1080/03091927708240383.

Edwards, D. C., and T. B. McKee, 1997:Characteristics of 20th century drought in the United States at multiple time scales. Climatology Rep. 97-2, Dept. of Atmospheric Science, Colorado State University, 155 pp., https://mountainscholar.org/handle/ 10217/170176.

Ellis, A. W., and N. P. Barton, 2012: Characterizing the North Pacific jet stream for understanding historical variability in western United States winter precipitation. Phys. Geogr., 33, 105-128, https://doi.org/10.2747/0272-3646.33.2.105.

Guttman, N. B., 1992: Spatial comparability of the Palmer Drought Severity Index. Water Res. Bull., 28, 1111-1119, https://doi.org/ 10.1111/j.1752-1688.1992.tb04022.x.

_ - 1998: Comparing the Palmer Drought Index and the Standardized Precipitation Index. J. Amer. Water Resour. Assoc., 34, 113-121, https://doi.org/10.1111/j.1752-1688.1998.tb05964.x.

Hannachi, A., I. T. Jolliffe, and D. B. Stephenson, 2007: Empirical orthogonal functions and related techniques in atmospheric science: A review. Int. J. Climatol., 27, 1119-1152, https:// doi.org/10.1002/joc.1499.

Heim, R. R., 2002: A review of twentieth-century drought indices used in the United States. Bull. Amer. Meteor. Soc., 83, 11491166, https://doi.org/10.1175/1520-0477-83.8.1149.

Kalnay, E., and Coauthors, 1996: The NCEP/NCAR 40-Year Reanalysis Project. Bull. Amer. Meteor. Soc., 77, 437-471, https://doi.org/10.1175/1520-0477(1996)077<0437:TNYRP> 2.0.CO;2.

Karl, T. R., 1983: Some spatial characteristics of drought duration in the United States. J. Climate Appl. Meteor., 22, 1356-1366, https://doi.org/10.1175/1520-0450(1983)022<1356:SSCODD> 2.0.CO;2.

L'Heureux, M. L., M. E. Mann, B. I. Cook, B. E. Gleason, and R. S. Vose, 2004: Atmospheric circulation influences on seasonal precipitation patterns in Alaska during the latter 20th century. J. Geophys. Res., 109, D06106, https://doi.org/10.1029/ 2003JD003845.

McKee, T. B., N. J. Doesken, and J. Kleist, 1993: The relationship of drought frequency and duration to time scales. Preprints, Eighth Conf. on Applied Climatology, Anaheim, CA, Amer. Meteor. Soc., 179-184.

Michaelsen, J., 1987: Cross-validation in statistical forecast models. J. Climate Appl. Meteor., 26, 1589-1600, https://doi.org/10.1175/ 1520-0450(1987)026<1589:CVISCF $>2.0 . \mathrm{CO} ; 2$.
Mitchell, T. P., and W. Blier, 1997: The variability of wintertime precipitation in the region of California. J. Climate, $\mathbf{1 0}$, 2261-2276, https://doi.org/10.1175/1520-0442(1997)010<2261: TVOWPI $>2.0 . \mathrm{CO} ; 2$.

Palmer, W. C., 1965: Meteorological drought. U.S. Weather Bureau Research Paper 45, 58 pp., http://www.ncdc.noaa.gov/ temp-and-precip/drought/docs/palmer.pdf.

Schnetzler, A. E., and C. F. Dierking, 2008: Seasonal temperature and precipitation dependencies in southeast Alaska. Natl. Wea. Dig., 32, 93-108.

Seager, R., M. Hoerling, S. Schubert, H. Wang, B. Lyon, A. Kumar, J. Nakamura, and N. Henderson, 2015: Causes of the 2011-14 California drought. J. Climate, 28, 6997-7024, https://doi.org/ 10.1175/JCLI-D-14-00860.1.

Swain, D. L., D. Singh, D. E. Horton, J. S. Mankin, T. C. Ballard, and N. S. Diffenbaugh, 2017: Remote linkages to anomalous winter atmospheric ridging over the northeastern Pacific. J. Geophys. Res. Atmos., 122, 12 194-12 209, https://doi.org/ 10.1002/2017JD026575.

Vicente-Serrano, S. M., S. Beguería, and J. I. López-Moreno, 2010: A multiscalar drought index sensitive to global warming: The standardized precipitation-evapotranspiration index. J. Climate, 23, 1696-1718, https://doi.org/10.1175/2009JCLI2909.1.

Wang, S.-Y., L. Hipps, R. R. Gillies, and J.-H. Yoon, 2014: Probable causes of the abnormal ridge accompanying the 2013-2014 California drought: ENSO precursors and anthropogenic warming footprint. Geophys. Res. Lett., $\mathbf{4 1}$, 3220-3226, https://doi.org/10.1002/2014GL059748.

Wells, N., S. Goddard, and M. J. Hayes, 2004: A self-calibrating Palmer drought severity index. J. Climate, 17, 2335-2351, https://doi.org/10.1175/1520-0442(2004)017<2335:ASPDSI> 2.0.CO;2.

Williams, A. P., B. I. Cook, J. E. Smerdon, D. A. Bishop, R. Seager, and J. S. Mankin, 2017: The 2016 southeastern U.S. drought: An extreme departure from centennial wetting and cooling. J. Geophys. Res. Atmos., 122, 10 888-10 905, https://doi.org/ 10.1002/2017JD027523.

Wu, R., and J. L. Kinter, 2009: Analysis of the relationship of U.S. droughts with SST and soil moisture: Distinguishing the time scale of droughts. J. Climate, 22, 4520-4538, https://doi.org/ 10.1175/2009JCLI2841.1.

Xia, Y., and Coauthors, 2012a: Continental-scale water and energy flux analysis and validation for the North American Land Data Assimilation System project phase 2 (NLDAS2): 1. Intercomparison and application of model products. J. Geophys. Res., 117, D03109, https://doi.org/10.1029/ 2011JD016048.

, and Coauthors, 2012b: Continental-scale water and energy flux analysis and validation for North American Land Data Assimilation System project phase 2 (NLDAS-2): 2. Validation of model-simulated streamflow. J. Geophys. Res., 117, D03110, https://doi.org/10.1029/2011JD016051. 